\title{
STRATIFIED COEFFICIENTS OF RELIABILITY AND THEIR SAMPLING BEHAVIOR UNDER NONNORMALITY
}

\author{
Haruhiko Ogasawara*
}

Stratified versions of coefficients for reliability are defined as extensions of the unstratified coefficients given by Guttman and Cronbach: Lambda 3 (Alpha), Lambda 2 and Lambda 6. One of the stratified coefficients is already available as stratified alpha. Among the four stratified coefficients dealt with in this article, two coefficients are for a stratified test with set (stratum)-specific true scores while the other two are for a stratified test with errors correlated within each set. Conditions of some coefficients being equal to population reliability are shown. For the sampling behavior, asymptotic distributions of the sample coefficients with and without stratification are derived using the asymptotic expansions under nonnormality with simulations for confirmation.

\section{Introduction}

In classical test theory, reliability of a composite score of a test with $p$ components is defined as $\rho_{X X^{\prime}}=\operatorname{var}(T) / \operatorname{var}(X)$, where $T=\sum_{i=1}^{p} T_{i}, \quad X=\sum_{i=1}^{p} X_{i}, \quad X_{i}\left(=T_{i}+E_{i}\right)$ is a continuous or discrete observed variable, $T_{i}$ is the true score of the $i$-th component, and $E_{i}$ is the corresponding measurement error. Note that the conventional notation $\rho_{X X^{\prime}}$ for reliability is used as a symbol even when a parallel form $X^{\prime}$ is not available. By definition $\operatorname{cov}\left(T_{i}, E_{j}\right)=0(i, j=1, \ldots, p)$ usually with the assumption, $\operatorname{cov}\left(E_{i}, E_{j}\right)=0(i \neq j)$. Since in a single test administration, only $X_{i}$ 's are observable, it is difficult to obtain $\rho_{X X^{\prime}}$ even when the $p \times p$ population covariance matrix $\boldsymbol{\Sigma}$ for $X_{i}$ 's is available without the information about the covariance structure of $T_{i}$ 's and $E_{i}$ 's. Let $\mathbf{x}=\left(X_{1}, \ldots, X_{p}\right)^{\prime}, \quad \mathbf{t}=\left(T_{1}, \ldots, T_{p}\right)^{\prime}$ and $\mathbf{e}=\left(E_{1}, \ldots, E_{p}\right)^{\prime}$ with $\operatorname{cov}\left(\mathbf{t}, \mathbf{e}^{\prime}\right)=O$. Then, $\boldsymbol{\Sigma}=\boldsymbol{\Gamma}+\boldsymbol{\Psi}$ with $\boldsymbol{\Gamma}=\operatorname{cov}(\mathbf{t})$ and $\boldsymbol{\Psi}=\operatorname{cov}(\mathbf{e})$, which gives

$$
\rho_{X X^{\prime}}=\frac{\mathbf{1}_{p}^{\prime} \boldsymbol{\Gamma} \mathbf{1}_{p}}{\mathbf{1}_{p}^{\prime} \boldsymbol{\Sigma} \mathbf{1}_{p}}=1-\frac{\mathbf{1}_{p}^{\prime} \boldsymbol{\Psi} \mathbf{1}_{p}}{\mathbf{1}_{p}^{\prime} \boldsymbol{\Sigma} \mathbf{1}_{p}},
$$

where $\mathbf{1}_{p}$ is the $p \times 1$ vector of 1 's.

When a factor analysis model $\boldsymbol{\Sigma}=\boldsymbol{\Lambda} \boldsymbol{\Lambda}^{\prime}+\boldsymbol{\Psi}_{\mathrm{D}}$ holds with a $p \times m(m<p)$ loading matrix $\boldsymbol{\Lambda}$ and a diagonal matrix $\boldsymbol{\Psi}_{\mathrm{D}}$ for unique factor (error) covariances, (1.1) becomes $\rho_{X X^{\prime}}=1-\left\{\mathbf{1}_{p}^{\prime} \mathbf{\Psi}_{\mathrm{D}} \mathbf{1}_{p} / \mathbf{1}_{p}^{\prime}\left(\boldsymbol{\Lambda} \boldsymbol{\Lambda}^{\prime}+\mathbf{\Psi}_{\mathrm{D}}\right) \mathbf{1}_{p}\right\}$. Coefficient $\omega$ using the factor analysis model was first given by McDonald (1970, Equation (A.17)) (see also McDonald, 1999, p.89 and Zinbarg, Revelle, Yovel \& Li, 2005). A sample counterpart $\hat{\rho}_{X X^{\prime}}$ of (1.1) is obtained by substituting the parameter estimates for the population values in (1.1). Practical estimation methods of $\rho_{X X^{\prime}}$ with factor analytic models using SEM (structural equation

Key Words and Phrases: reliability, Cronbach's alpha, stratification, Edgeworth expansion, Studentized estimators, nonnormality

* Department of Information and Management Science, Otaru University of Commerce, 3-5-21, Midori, Otaru 047-8501 Japan. E-mail: hogasa@res.otaru-uc.ac.jp

Partially supported by Grant-in-Aid for Scientific Research from the Japanese Ministry of Education, Culture, Sports, Science and Technology, No.20500249. 
modeling) software are given by Raykov (1997b) and Raykov and Shrout (2002). The asymptotic distributions of $\hat{\rho}_{X X^{\prime}}$ with the factor analytic models have been given by Raykov (2002) (see also, Raykov \& Marcoulides, 2004), and Yuan and Bentler (2002). Note that the above asymptotic distributions of $\hat{\rho}_{X X^{\prime}}$ with the factor analytic models are given when the model is true. In practice, usually the model may be more or less misspecified even when the model is not rejected by some statistical tests. When the misspecification is not negligible, asymptotic distributions of $\hat{\rho}_{X X^{\prime}}$ should be given from those of the parameter estimators with model misspecification (e.g., Ogasawara, 2007a) and from $\mathbf{S}$ in place of $\hat{\mathbf{\Lambda}} \hat{\boldsymbol{\Lambda}}^{\prime}+\hat{\mathbf{\Psi}}_{\mathrm{D}}$, where $\mathbf{S}$ is the usual unbiased sample covariance matrix corresponding to $\boldsymbol{\Sigma}^{*}=\mathrm{E}(\mathbf{S})\left(\neq \boldsymbol{\Lambda} \boldsymbol{\Lambda}^{\prime}+\boldsymbol{\Psi}_{\mathrm{D}}\right)$. That is, from (1.1),

$$
\operatorname{avar}\left(\hat{\rho}_{X X^{\prime}}\right)=\left.\left.\frac{\partial \hat{\rho}_{X X^{\prime}}}{\partial \mathbf{s}^{\prime}}\right|_{\mathbf{s}=\boldsymbol{\sigma}^{*}} \operatorname{acov}(\mathbf{s}) \frac{\partial \hat{\rho}_{X X^{\prime}}}{\partial \mathbf{s}}\right|_{\mathbf{s}=\boldsymbol{\sigma}^{*}}
$$

with

$$
\left.\frac{\partial \hat{\rho}_{X X^{\prime}}}{\partial \mathbf{s}^{\prime}}\right|_{\mathbf{s}=\boldsymbol{\sigma}^{*}}=-\left.\mathbf{1}_{p}^{\prime} \frac{\partial \hat{\mathbf{\Psi}}_{\mathrm{D}} \mathbf{1}_{p}}{\partial \mathbf{s}^{\prime}}\right|_{\mathbf{s}=\boldsymbol{\sigma}^{*}} \frac{1}{\mathbf{1}_{p}^{\prime} \boldsymbol{\Sigma}^{*} \mathbf{1}_{p}}+\frac{\mathbf{1}_{p}^{\prime} \mathbf{\Psi}_{\mathrm{D}} \mathbf{1}_{p}}{\left(\mathbf{1}_{p}^{\prime} \boldsymbol{\Sigma}^{*} \mathbf{1}_{p}\right)^{2}} \mathbf{1}_{p^{2}}^{\prime} \mathbf{D}_{p},
$$

where $\operatorname{avar}(\cdot)$ is the asymptotic variance of the $\operatorname{argument}$ estimator; $\operatorname{acov}(\cdot)$ is the asymptotic covariance matrix of the argument estimators; $\mathbf{s}=\mathrm{v}(\mathbf{S}) ; \boldsymbol{\sigma}^{*}=\mathrm{v}\left(\boldsymbol{\Sigma}^{*}\right) ; \mathrm{v}(\cdot)$ denotes the vectorizing operator taking the nonduplicated elements of a symmetric matrix; and $\mathbf{D}_{p}$ is the duplication matrix with $\operatorname{vec}(\mathbf{S})=\mathbf{D}_{p} \mathrm{v}(\mathbf{S})$, where $\operatorname{vec}(\cdot)$ is an operator that vectorizes a matrix by stacking its columns. Note that the partial derivative $\partial \hat{\mathbf{\Psi}}_{\mathrm{D}} \mathbf{1}_{p} / \partial \mathbf{s}^{\prime}$ in (1.3) is given by using the property that $\hat{\mathbf{\Psi}}_{\mathrm{D}}=\mathbf{\Psi}_{\mathrm{D}}(\mathbf{s})$ is an implicit function of $\mathbf{s}$ (see Ogasawara, 2007a) as in the case without model misspecification. When $\mathbf{1}_{p}^{\prime} \hat{\boldsymbol{\Sigma}} \mathbf{1}_{p}$ with $\hat{\boldsymbol{\Sigma}}=\hat{\boldsymbol{\Lambda}} \hat{\boldsymbol{\Lambda}}^{\prime}+\hat{\mathbf{\Psi}}_{\mathrm{D}}$ is used for the estimator of $\mathbf{1}_{p}^{\prime} \boldsymbol{\Sigma}^{*} \mathbf{1}_{p}$ irrespective of misspecification, $\boldsymbol{\Sigma}^{*}$ and $\mathbf{1}_{p^{2}}^{\prime} \mathbf{D}_{p}$, in (1.3) should be replaced by $\boldsymbol{\Sigma}\left(=\boldsymbol{\Lambda} \boldsymbol{\Lambda}^{\prime}+\mathbf{\Psi}_{\mathrm{D}}\right)$ and $\mathbf{1}_{p^{2}}^{\prime} \mathbf{D}_{p} \partial \hat{\boldsymbol{\sigma}} /\left.\partial \mathbf{s}^{\prime}\right|_{\mathbf{s}=\boldsymbol{\sigma}^{*}}$, respectively, where $\hat{\boldsymbol{\sigma}}=\mathrm{v}(\hat{\boldsymbol{\Sigma}})$. The normal approximation of the distribution of $\hat{\rho}_{X X^{\prime}}$ using (1.2) with (1.3) is asymptotically valid. However, even in large samples, $\hat{\rho}_{X X^{\prime}}$ may be grossly different from the population reliability $\rho_{X X^{\prime}}$ when the model misspecification is substantial.

The coefficients without model fitting, as approximations to reliability, have also been proposed in various ways (see Guttman, 1945; Feldt \& Brennan, 1989; Osburn, 2000). Among these coefficients, Cronbach's (1951) coefficient $\alpha$ has been one of the most frequently used ones which is defined as

$$
\alpha=\frac{p}{p-1}\left(1-\frac{\mathbf{1}_{p}^{\prime} \operatorname{Diag}(\boldsymbol{\Sigma}) \mathbf{1}_{p}}{\mathbf{1}_{p}^{\prime} \boldsymbol{\Sigma} \mathbf{1}_{p}}\right),
$$

where $\operatorname{Diag}(\cdot)$ denotes the diagonal matrix with the same diagonal elements as those of the argument matrix. It is known that $\alpha$ is equivalent to Guttman's $\lambda_{3}$ and functionally equivalent to various preceding coefficients (see Novick \& Lewis, 1967, p.1; Komaroff, 1997, p.337). The exact distribution of the estimator $\hat{\alpha}$ of $\alpha$ was given by Kristof (1963) and Feldt (1965) under the assumption of compound symmetry for $\boldsymbol{\Sigma}$ (i.e., equal variances and equal covariances) and multivariate normality for $\mathbf{x}$ (see also Feldt, Woodruff $\&$ Salih, 1987). The above restrictive form of $\boldsymbol{\Sigma}$ was relaxed under normality by van Zyl, 
Neudecker and Nel (2000). The exact distribution of $\hat{\alpha}$ with general $\boldsymbol{\Sigma}$ under normality was derived by Kistner and Muller (2004). Yuan and Bentler (2002) showed that the normal-theory (NT) asymptotic variance of $\hat{\alpha}$ holds for some nonnormal cases. Coefficient $\alpha$ can also be used for standardized variables, which gives the so-called standardized $\alpha$ (do not confuse with the standardization of $\hat{\alpha}$ using its standard error given later). The asymptotic distribution of the estimator of standardized $\alpha$ under normality was provided by Hayashi and Kamata (2005). The assumption of normality was removed by Ogasawara (2006b) for the estimators of $\alpha$ with and without standardization for observed variables and with fixed weights for standardized observed variables. Ogasawara's results under nonnormality were asymptotically more accurate than the usual normal approximation of the distribution of $\hat{\alpha}$ as used in Yuan and Bentler (2002).

It is known that $\alpha$ is a lower bound to $\rho_{X X^{\prime}}$ with the assumption of uncorrelated errors (Guttman, 1945) and that $\alpha=\rho_{X X^{\prime}}$ if and only if essential tau-equivalence is satisfied (i.e., the covariances and variances of $T_{i}$ 's are equal; see e.g., Novick \& Lewis, 1967). Raykov (1997a, Table 1) showed that in many cases of $\boldsymbol{\Sigma}$ encountered in practice, $\alpha$ is a good approximation to $\rho_{X X^{\prime}}$. It is, however, also known that when the errors $\mathbf{e}$ are positively correlated, $\alpha$ can give values greater than $\rho_{X X^{\prime}}$ (Zimmerman, Zumbo \& Lalonde, 1993; Komaroff, 1997; Raykov, 1998, 2001). It is to be noted that when the errors are negatively correlated, though unusual in practice, $\alpha$ becomes smaller than that with uncorrelated errors (see Raykov, 2001, Table 1).

From (1.4), coefficient $\alpha$ is seen as the ratio of the average of the $p^{2}-p$ off-diagonal elements of $\boldsymbol{\Sigma}$ to that of all $p^{2}$ elements of $\boldsymbol{\Sigma}$. The former average of the off-diagonal elements is also seen as the average of all $p^{2}$ elements of $\boldsymbol{\Sigma}$ in which the diagonal elements are replaced by the average of the $p^{2}-p$ off-diagonal elements. Note that the substituted diagonal elements correspond to the variances of true scores approximated by (1.4) even in the population, whose approximation is not always accurate. Consider the situations of the following $\boldsymbol{\Sigma}$ 's with $p=4$ :

$$
\boldsymbol{\Sigma}_{\mathrm{A}}=\left[\begin{array}{llll}
1 & \multicolumn{2}{c}{\text { sym. }} \\
.4 & 1 & & \\
.4 & .4 & 1 & \\
.4 & .4 & .4 & 1
\end{array}\right] \text { and } \boldsymbol{\Sigma}_{\mathrm{B}}=\left[\begin{array}{llll}
1 & \text { sym. } \\
.8 & 1 & & \\
.2 & .2 & 1 & \\
.2 & .2 & .8 & 1
\end{array}\right]
$$

In these cases, we have the common value of $\alpha=.4 / .55 \doteq .73$, where .4 and .55 are the averages of the off-diagonal and all elements of $\boldsymbol{\Sigma}$ 's, respectively. In the case of $\boldsymbol{\Sigma}_{\mathrm{A}}$, the value .4 , as an approximation to the variances of true scores, may be reasonable (we do not consider the case where the variances of specific factors in factor analysis are part of the variances of true scores). In the second case of $\boldsymbol{\Sigma}_{\mathrm{B}}$, the four variables are composed of two groups with high within-group covariances and low between-group covariances. With such grouping or stratification, the approximation of the variances of true scores by .4 may not be reasonable. If we consider that the high within-group covariances stem from the existence of group-specific true scores, .8 is an alternative approximation. On the other hand, if the high covariances are due to the errors correlated within groups, .2 is more appropriate than .8. In addition, the within-group covariances .8 in $\boldsymbol{\Sigma}_{\mathrm{B}}$ are to be replaced 
by .2 when the covariances are used as those of true scores. In the two extreme cases, the modified coefficients (stratified coefficients $\lambda_{3 S}$ and $\lambda_{3 \mathrm{~T}}$ given in the next section) become

$$
1-\frac{2 \times 2.8\{1-(.4 / .7)\}}{8.8}=\frac{.50}{.55} \doteq .91 \text { and } \frac{.20}{.55} \doteq .36,
$$

respectively, which are substantially different from $\alpha \doteq .73$. In the following sections, the stratified coefficients of reliability will be defined with the derivation of the asymptotic distributions of the estimators under nonnormality.

\section{Stratified coefficients of reliability}

Denote $\alpha$ by $\lambda_{3}$ (Guttman, 1945) for convenience of notation with comparison to other coefficients. The stratified version of $\lambda_{3}$ denoted by $\lambda_{3 \mathrm{~S}}\left(=\alpha_{\mathrm{S}}\right)$ was introduced by Cronbach, Schönemann and McKie (1965) (see also Rajaratnam, Cronbach \& Gleser, 1965) when a test is comprised of sets (strata) of components. A set or a stratum may be a subtest in a test, a test battery in a set of test batteries and so on. Let $B$ be the number of the sets (strata) with $p_{I}$ being the number of components in the $I$-th set $(I=1, \ldots, B)$ and $\sum_{I=1}^{B} p_{I}=p$. Then, $\lambda_{3 \mathrm{~S}}$ is given by

$$
\lambda_{3 \mathrm{~S}}=1-\frac{\sum_{I=1}^{B} \sigma_{I}^{2}\left(1-\lambda_{3(I)}\right)}{\sigma^{2}}
$$

(Feldt \& Brennan, 1989, Equation (28)), where $\sigma_{I}^{2}=\operatorname{var}\left(\mathbf{1}_{p_{I}}^{\prime} \mathbf{x}_{(I)}\right) ; \mathbf{x}=\left(\mathbf{x}_{(1)}^{\prime}, \ldots, \mathbf{x}_{(B)}^{\prime}\right)^{\prime}$; $\mathbf{x}_{(I)}=\left(X_{I_{1}}, \ldots, X_{I_{p_{I}}}\right)^{\prime}$ is the vector of $p_{I}$ observed variables in the $I$-th set with $I_{j}=\sum_{J=1}^{I} p_{J}-p_{I}+j\left(j=1, \ldots, p_{I}\right) ; \sigma^{2}=\operatorname{var}(X)=\mathbf{1}_{p}^{\prime} \boldsymbol{\Sigma} \mathbf{1}_{p}$; and $\lambda_{3(I)}$ is $\lambda_{3}$ given by only $\mathbf{x}_{(I)}$. It is easily seen that $\sigma_{I}^{2}\left(1-\lambda_{3(I)}\right)$ is an upper bound to $\operatorname{var}\left(\mathbf{1}_{p_{I}}^{\prime} \mathbf{e}_{(I)}\right)$, when errors are uncorrelated, with $\mathbf{x}_{(I)}=\mathbf{t}_{(I)}+\mathbf{e}_{(I)}, \mathbf{t}_{(I)}$ and $\mathbf{e}_{(I)}$ defined for the $I$-th set similarly to $\mathbf{t}$ and $\mathbf{e}$. It is easy to find that when $B=1,(2.1)$ reduces to $\lambda_{3}$. The advantages of $\lambda_{3 \mathrm{~S}}$ (illustrated earlier when the high within-set covariances are due to set-specific true scores) are also shown by e.g., Cronbach (1988) and Osburn (2000).

Using (1.1) for $\lambda_{3(I)},(2.1)$ is equivalently expressed by

$$
\lambda_{3 \mathrm{~S}}=\left(\sum_{I \neq J} \sigma_{I_{i} J_{j}}+\sum_{I=1}^{B} \sigma_{I}^{2} \lambda_{3(I)}\right) / \sigma^{2}=\left(\sum_{I \neq J} \sigma_{I_{i} J_{j}}+\sum_{I=1}^{B} \frac{p_{I}}{p_{I}-1} \sum_{i \neq j} \sigma_{I_{i} I_{j}}\right) / \sigma^{2}
$$

where $\sigma_{I_{i} J_{j}}=\operatorname{cov}\left(X_{I_{i}}, X_{J_{j}}\right)$; and $\sum_{I \neq J}$ and $\sum_{i \neq j} \operatorname{denote} \sum_{\substack{I=1 \\ I \neq J}}^{B} \sum_{J=1}^{B} \sum_{i=1}^{p_{I}} \sum_{j=1}^{p_{J}}$ and $\sum_{\substack{i=1 \\ i \neq j}}^{p_{I}} \sum_{j=1}^{p_{I}}$, respectively. The numerator on the right-hand side of (2.2) is a lower bound to $\mathbf{1}_{p}^{\prime} \boldsymbol{\Gamma} \mathbf{1}_{p}$ with uncorrelated errors (recall (1.1)), where the diagonal elements of $\boldsymbol{\Gamma}$ in the $I$-th set are reproduced by the mean of $\sigma_{I_{i} I_{j}}(i \neq j)$. Note that $\lambda_{3}$ neglecting stratification is

$$
\lambda_{3}=\frac{p}{p-1} \sum_{i \neq j} \sigma_{i j} / \sigma^{2}
$$

(see (1.4)), where the diagonal elements of $\boldsymbol{\Gamma}$ are reproduced by the mean of all the cross 
covariances $\sigma_{i j}(i, j=1, \ldots, p ; i \neq j)$ including $\sigma_{I_{i} J_{j}}(I \neq J)$ in (2.2). From (2.2) and (2.3) with this interpretation, it is seen that when observed variables are correlated within each set more strongly than between sets, $\lambda_{3 \mathrm{~S}}$ becomes larger than $\lambda_{3}$. For the relationship of $\lambda_{3 S}$ and $\rho_{X X^{\prime}}$, the following result is obtained.

Theorem 1. $\lambda_{3 S}=\rho_{X X^{\prime}}$ if and only if each set of observed variables $\mathbf{x}_{(I)}(I=1, \ldots, B)$ satisfies essential tau-equivalence when errors are uncorrelated within and between sets.

Proof. From (1.1) and (2.2) with the assumption of uncorrelated errors, noting $\gamma_{I_{i} I_{j}}=(\boldsymbol{\Gamma})_{I_{i} I_{j}}=\sigma_{I_{i} I_{j}}(i \neq j)$ with $(\cdot)_{I_{i} I_{j}}$ being the $\left(I_{i}, I_{j}\right)$ th element of the argument matrix, it follows that

$$
\begin{aligned}
\sigma^{2}\left(\rho_{X X^{\prime}}-\lambda_{3 \mathrm{~S}}\right) & =\sum_{I=1}^{B} \sum_{i=1}^{p_{I}} \gamma_{I_{i} I_{i}}-\sum_{I=1}^{B} \frac{1}{p_{I}-1} \sum_{i \neq j} \gamma_{I_{i} I_{j}} \\
& =\sum_{I=1}^{B} \frac{1}{2\left(p_{I}-1\right)} \sum_{i \neq j} \operatorname{var}\left(T_{I_{i}}-T_{I_{j}}\right) \geq 0 .
\end{aligned}
$$

The equality holds if and only if $T_{I_{1}}=\cdots=T_{I_{p_{I}}}(I=1, \ldots, B)$ except for additive constants (i.e., essential tau-equivalence holds). Q. E. D.

It is apparent that Theorem 1 gives the condition weaker than that of the full tauequivalence without stratification. Consequently, it is expected that $\lambda_{3 S}$ tends to give a lower bound closer to $\rho_{X X^{\prime}}$ than $\lambda_{3}$. The disadvantage of the inflated value of $\lambda_{3}$ with positively correlated errors can also be reduced in typical cases. Consider the situation of a set of two alternative forms $(B=2)$, which are composed of similar components $\mathbf{x}_{(1)}$ and $\mathbf{x}_{(2)}$ with $p_{1}=p_{2}=p^{*}(=p / 2)$, say if not parallel (see the similar situation considered by Raykov, 1997a for criticism of $\left.\lambda_{3}\right)$. Let $\operatorname{cov}\left(E_{1_{i}}, E_{2_{i}}\right)=\psi_{12 i}>0\left(i=1, \ldots, p^{*}\right)$ with $\psi_{12 i}=\left(\boldsymbol{\Psi}_{12}\right)_{i i}$ and $\boldsymbol{\Psi}=\left[\begin{array}{ll}\boldsymbol{\Psi}_{11} & \boldsymbol{\Psi}_{12} \\ \boldsymbol{\Psi}_{21} & \boldsymbol{\Psi}_{22}\end{array}\right]$, and let other error covariances be 0. That is, the errors of the corresponding components in the two forms are supposed to be positively correlated, which often happens in practice. Let $\boldsymbol{\Gamma}$ be given by essential tau-equivalence i.e., $\boldsymbol{\Gamma}=\gamma^{*} \mathbf{1}_{p} \mathbf{1}_{p}^{\prime}$ with $\gamma^{*}>0$ for fair comparison to $\lambda_{3}$. That is, without the positive correlations of the errors,

$$
\lambda_{3}=\lambda_{3 \mathrm{~S}}=\rho_{X X^{\prime}}=\frac{p^{2} \gamma^{*}}{p^{2} \gamma^{*}+\sum_{i=1}^{p}(\mathbf{\Psi})_{i i}},
$$

while with the positive correlations of the errors,

$$
\lambda_{3}=\frac{p^{2} \gamma^{*}+\{2 p /(p-1)\} \sum_{i=1}^{p *} \psi_{12 i}}{p^{2} \gamma^{*}+\sum_{i=1}^{p}(\Psi)_{i i}+2 \sum_{i=1}^{p^{*}} \psi_{12 i}}>\frac{p^{2} \gamma^{*}+2 \sum_{i=1}^{p^{*}} \psi_{12 i}}{p^{2} \gamma^{*}+\sum_{i=1}^{p}(\boldsymbol{\Psi})_{i i}+2 \sum_{i=1}^{p^{*}} \psi_{12 i}}=\lambda_{3 \mathrm{~S}}>\rho_{X X^{\prime}}
$$

where the same notation $\lambda_{3}, \lambda_{3 S}$ and $\rho_{X X^{\prime}}$ are used for simplicity irrespective of different error correlations. Note that the reliability of (2.6) is smaller than that of (2.5) due to the positive error correlations (if $\sum_{i=1}^{p^{*}} \psi_{12 i}$ is negative, the directional reversal of the inequality signs in (2.6) follows and $\rho_{X X^{\prime}}$ in (2.5) becomes smaller than that of (2.6)).

Although the above example shows an advantage of $\lambda_{3 S}$ (i.e., $\lambda_{3 S}$ is closer to $\rho_{X X^{\prime}}$ than 
$\lambda_{3}$ ), a counter example can be easily constructed. Use the above situation with different correlations of errors such that

$$
\boldsymbol{\Psi}=\boldsymbol{\Psi}_{0}+\psi_{0}\left[\begin{array}{lc}
\mathbf{1}_{p *} \mathbf{1}_{p *}^{\prime} & O \\
O & \mathbf{1}_{p *} \mathbf{1}_{p *}^{\prime}
\end{array}\right], \psi_{0}>0, \quad\left(\boldsymbol{\Psi}_{0}\right)_{i j}=0(i \neq j) .
$$

That is, $\mathbf{e}$ is correlated only within each set. In this case, recalling $p^{*}=p_{1}=p_{2}=p / 2$,

$$
\lambda_{3}=\frac{p^{2} \gamma^{*}+\left\{2 p\left(p^{* 2}-p^{*}\right) /(p-1)\right\} \psi_{0}}{p^{2} \gamma^{*}+\sum_{i=1}^{p}\left(\mathbf{\Psi}_{0}\right)_{i i}+\left(p^{2} / 2\right) \psi_{0}}>\frac{p^{2} \gamma^{*}}{p^{2} \gamma^{*}+\sum_{i=1}^{p}\left(\mathbf{\Psi}_{0}\right)_{i i}+\left(p^{2} / 2\right) \psi_{0}}=\rho_{X X^{\prime}}
$$

while

$$
\lambda_{3 \mathrm{~S}}=\frac{p^{2} \gamma^{*}+\left(p^{2} / 2\right) \psi_{0}}{p^{2} \gamma^{*}+\sum_{i=1}^{p}\left(\mathbf{\Psi}_{0}\right)_{i i}+\left(p^{2} / 2\right) \psi_{0}}>\lambda_{3}>\rho_{X X^{\prime}}
$$

since

$$
\left\{\frac{2 p\left(p^{* 2}-p^{*}\right)}{p-1}-\frac{p^{2}}{2}\right\} \psi_{0}=-\frac{p^{2} \psi_{0}}{2(p-1)}<0 .
$$

Noting that without the within-set positive correlations of errors $\lambda_{3 \mathrm{~S}}=\lambda_{3}=\rho_{X X^{\prime}}$, (2.9) shows a disadvantage of $\lambda_{3 \mathrm{~S}}$. However, the added term $\psi_{0}[\cdot]$ on the right-hand side of the first equation of (2.7) may also be seen as the contribution of the battery (form)-specific factors in inter-battery factor analysis (Tucker, 1958; Wegelin, Packer \& Richardson, 2006). Whether the specific factors are to be regarded as errors or true scores depends on definitions/conceptualizations (see Zimmerman et al., 1993, p.45; Zinbarg et al., 2005, p.126). The contribution of the specific factors can be experimentally confirmed by e.g., the test-retest method (Jöreskog \& Sörbom, 1976). When the term $\psi_{0}[\cdot]$ is seen as given by part of true scores with $\boldsymbol{\Sigma}$ unchanged, it follows from Theorem 1 that $\lambda_{3 \mathrm{~S}}=\rho_{X X^{\prime}}>\lambda_{3}$ with $\operatorname{cor}\left(T_{1 i}, T_{2 j}\right)=\gamma^{*} /\left(\gamma^{*}+\psi_{0}\right)<1\left(i, j=1, \ldots, p^{*}\right)$, which were formerly 1 .

In the case of (2.7) with correlated errors within each set (not part of true scores), the following new coefficient (between-set $\lambda_{3}$ ) may be used:

$$
\lambda_{3 \mathrm{~T}}=\frac{p^{2}}{p^{2}-\sum_{I=1}^{B} p_{I}^{2}} \sum_{I \neq J} \sigma_{I_{i} J_{j}} / \sigma^{2}
$$

which has the following property whose derivation can be easily given.

Theorem 2. $\lambda_{3 \mathrm{~T}}=\rho_{X X^{\prime}}$ if essential tau-equivalence holds for all sets of variables with errors uncorrelated between sets and possibly correlated within sets.

Note that the necessity does not hold, which can be explained as follows. Let $T_{I}=\mathbf{1}_{p_{I}}^{\prime} \mathbf{t}_{(I)}=\sum_{i=1}^{p_{I}} T_{I_{i}}$. Then, the scaled difference of $\rho_{X X^{\prime}}$ and $\lambda_{3 \mathrm{~T}}$ is

$$
\begin{gathered}
\sigma^{2}\left(\rho_{X X^{\prime}}-\lambda_{3 \mathrm{~T}}\right)=\sum_{I=1}^{B} \sum_{i=1}^{p_{I}} \sum_{j=1}^{p_{I}} \gamma_{I_{i} I_{j}}-\frac{\sum_{I=1}^{B} p_{I}^{2}}{p^{2}-\sum_{I=1}^{B} p_{I}^{2}} \sum_{I \neq J} \gamma_{I_{i} J_{j}} \\
=\sum_{I=1}^{B} \operatorname{var}\left(T_{I}\right)-\frac{\sum_{I=1}^{B} p_{I}^{2}}{p^{2}-\sum_{I=1}^{B} p_{I}^{2}} \sum_{I \neq J} \operatorname{cov}\left(T_{I}, T_{J}\right)
\end{gathered}
$$




$$
\begin{aligned}
& =\left(1-\frac{(B-1) \sum_{I=1}^{B} p_{I}^{2}}{p^{2}-\sum_{I=1}^{B} p_{I}^{2}}\right) \sum_{I=1}^{B} \operatorname{var}\left(T_{I}\right)+\frac{\sum_{I=1}^{B} p_{I}^{2}}{2\left(p^{2}-\sum_{I=1}^{B} p_{I}^{2}\right)} \sum_{I \neq J} \operatorname{var}\left(T_{I}-T_{J}\right) \\
& =\frac{-B \sum_{I=1}^{B}\left(p_{I}-B^{-1} \sum_{J=1}^{B} p_{J}\right)^{2}}{p^{2}-\sum_{I=1}^{B} p_{I}^{2}} \sum_{I=1}^{B} \operatorname{var}\left(T_{I}\right)+\frac{\sum_{I=1}^{B} p_{I}^{2}}{2\left(p^{2}-\sum_{I=1}^{B} p_{I}^{2}\right)} \sum_{I \neq J} \operatorname{var}\left(T_{I}-T_{J}\right),
\end{aligned}
$$

where the first term on the right-hand side of the last equation becomes zero when $p_{1}=\cdots=p_{B}$ while the second term vanishes when $T_{1}=\cdots=T_{B}$. However, the last case does not necessarily mean the essential tau-equivalence for all sets of variables. From (2.12), we find that $\lambda_{3 \mathrm{~T}}$ gives a lower bound of $\rho_{X X^{\prime}}$ when $p_{1}=\cdots=p_{B}$ with the assumption of Theorem 2 for errors. This finding suggests a modified $\lambda_{3 \mathrm{~T}}$ given by

$$
\lambda_{3 \mathrm{~T}^{*}}=\frac{B}{B-1} \sum_{I \neq J} \sigma_{T_{I} T_{J}} / \sigma^{2} \quad \text { with } \quad \sigma_{T_{I} T_{J}}=\operatorname{cov}\left(T_{I}, T_{J}\right),
$$

which always gives a lower bound of $\rho_{X X^{\prime}}$ irrespective of $p_{1} \neq p_{J}$ for some $I$ and $J$ with the assumption of Theorem 2 for errors. It is obvious that $\lambda_{3 \mathrm{~T}^{*}}$ reduces to $\lambda_{3}$ if $T_{I}$ 's are redefined as $T_{i}$ 's in a single set of variables with $p=B$.

From Theorem 2, in the case of (2.7) with correlated errors within sets, $\lambda_{3 \mathrm{~T}}=\rho_{X X^{\prime}}<$ $\lambda_{3}<\lambda_{3 \mathrm{~S}}$. Note that when errors are correlated across sets as in the case with (2.5) and (2.6), $\lambda_{3 \mathrm{~T}}$ gives inflated values. In these cases, the two sets can be replaced by new $p^{*}$ sets with two corresponding components $X_{1_{i}}$ and $X_{2}{ }_{i}\left(i=1, \ldots, p^{*}\right)$ as new members of each new set. This gives again $\lambda_{3 \mathrm{~T}}=\rho_{X X^{\prime}}$.

\section{Applications to other coefficients}

The formula of (2.1) can also be used for other coefficients. It is known that Guttman's (1945) $\lambda_{2}$ is a better lower bound to $\rho_{X X^{\prime}}$, when errors are uncorrelated, in the sense

$$
\lambda_{3} \leq \lambda_{2}=\left\{\sum_{i \neq j} \sigma_{i j}+\left(\frac{p}{p-1} \sum_{i \neq j} \sigma_{i j}^{2}\right)^{1 / 2}\right\} / \sigma^{2} \leq \rho_{X X^{\prime}}
$$

(see e.g., Lord \& Novick, 1968, Theorem 4.4.7). Ten Berge and Zegers (1978) derived an infinite series $\lambda_{3}, \lambda_{2}, \ldots$ such that $\lambda_{3} \leq \lambda_{2} \leq \cdots \leq \rho_{X X^{\prime}}$. Since the series does not necessarily converge to $\rho_{X X^{\prime}}$ for some $\boldsymbol{\Sigma}$ and the increases beyond $\lambda_{2}$ are, in most cases, not substantial, $\lambda_{2}$ may be only the coefficient worth computing beyond $\lambda_{3}$ in the series. The two inequalities in (3.1) become equalities simultaneously if and only if essential tau-equivalence holds.

Stratified $\lambda_{2}\left(\lambda_{2 \mathrm{~S}}\right)$ is defined similarly to $\lambda_{3 \mathrm{~S}}$ :

$$
\lambda_{2 \mathrm{~S}}=\left[\sum_{I \neq J} \sigma_{I_{i} J_{j}}+\sum_{I=1}^{B}\left\{\sum_{i \neq j} \sigma_{I_{i} I_{j}}+\left(\frac{p_{I}}{p_{I}-1} \sum_{i \neq j} \sigma_{I_{i} I_{j}}^{2}\right)^{1 / 2}\right\}\right] / \sigma^{2} .
$$

It is obvious that the result similar to Theorem 1 holds for $\lambda_{2 S}$ using the condition as for 
$\lambda_{2}=\rho_{X X^{\prime}}$.

Guttman's (1945) $\lambda_{6}$ is a coefficient based on the residual variance in multiple regression analysis:

$$
\begin{aligned}
\lambda_{6} & =1-\left\{\sum_{i=1}^{p}\left(\sigma_{i i}-\boldsymbol{\sigma}_{(i)}^{\prime} \boldsymbol{\Sigma}_{(i i)}^{-1} \boldsymbol{\sigma}_{(i)}\right) / \sigma^{2}\right\} \\
& =1-\left\{\sum_{i=1}^{p}\left(\sigma^{i i}\right)^{-1} / \sigma^{2}\right\}=1-\left\{\sum_{i=1}^{p} \sigma_{i i}\left(1-\rho_{(i)}^{2}\right) / \sigma^{2}\right\},
\end{aligned}
$$

where $\boldsymbol{\sigma}_{(i)}$ is the $(p-1) \times 1$ vector consisting of the elements of the $i$-th column of $\boldsymbol{\Sigma}$ omitting $\sigma_{i i} ; \boldsymbol{\Sigma}_{(i i)}$ is the $(p-1) \times(p-1)$ matrix given by $\boldsymbol{\Sigma}$ deleting the $i$-th column and the $i$-th row; $\sigma^{i j}=\left(\boldsymbol{\Sigma}^{-1}\right)_{i j}$; and $\rho_{(i)}^{2}$ is the squared multiple correlation for the $i$-th variable regressed on the remaining ones. It is known that $\lambda_{6} \leq \rho_{X X^{\prime}}$ (see e.g., Guttman, 1945, p.278). Since there is no ordering between $\lambda_{3}\left(\lambda_{2}\right)$ and $\lambda_{6}$ as between $\lambda_{3}$ and $\lambda_{2}$, $\lambda_{6}$ has its own advantage (see Woodhouse \& Jackson, 1977, Table 4.3). The between-set $\lambda_{6}\left(\lambda_{6 \mathrm{~T}}\right)$ using $B$ sets is defined as

$$
\begin{aligned}
\lambda_{6 \mathrm{~T}} & =1-\left\{\sum_{M=1}^{B} \mathbf{1}_{p_{M}}^{\prime}\left(\boldsymbol{\Sigma}_{M M}-\boldsymbol{\Sigma}_{M(M)} \boldsymbol{\Sigma}_{(M M)}^{-1} \boldsymbol{\Sigma}_{(M) M}\right) \mathbf{1}_{p_{M}} / \sigma^{2}\right\} \\
& =1-\left\{\sum_{M=1}^{B} \mathbf{1}_{p_{M}}^{\prime}\left(\boldsymbol{\Sigma}^{M M}\right)^{-1} \mathbf{1}_{p_{M}} / \sigma^{2}\right\},
\end{aligned}
$$

where $\boldsymbol{\Sigma}_{M M}$ is the $p_{M} \times p_{M}$ matrix equal to the $M$-th diagonal block of $\boldsymbol{\Sigma}$ for the $M$-th set; $\boldsymbol{\Sigma}_{M(M)}\left(=\boldsymbol{\Sigma}_{(M) M}^{\prime}\right)$ is the $p_{M} \times\left(p-p_{M}\right)$ matrix consisting of the rows of $\boldsymbol{\Sigma}$ for the $M$-th set omitting the columns for the $M$-th set; $\boldsymbol{\Sigma}_{(M M)}$ is the $\left(p-p_{M}\right) \times\left(p-p_{M}\right)$ matrix without the rows and columns for the $M$-th set; and $\boldsymbol{\Sigma}^{M M}$ is the $p_{M} \times p_{M}$ matrix equal to the $M$-th diagonal block of $\boldsymbol{\Sigma}^{-1}$. Note that $\lambda_{6 \mathrm{~S}}$ is not defined in this article and that $\lambda_{6} \geq \lambda_{6 \mathrm{~T}}$ due to the property of regression.

So far, $\lambda_{3}(=\alpha), \lambda_{3 \mathrm{~S}}, \lambda_{3 \mathrm{~T}}\left(\lambda_{3 \mathrm{~T}^{*}}\right), \lambda_{2}, \lambda_{2 \mathrm{~S}}, \lambda_{6}$ and $\lambda_{6 \mathrm{~T}}$ are defined, where to the author's knowledge $\lambda_{3 \mathrm{~T}}\left(\lambda_{3 \mathrm{~T}^{*}}\right), \lambda_{2 \mathrm{~S}}$ and $\lambda_{6 \mathrm{~T}}$ are new. As addressed in Section 1, when observed variables are standardized, the coefficients can be defined using the correlation matrix. The cases with fixed weights for standardized variables are considered (note that the corresponding results for unstandardized variables reduce to the cases with unstandardized variables with weighted variables redefined as unweighted ones).

Let $\mathbf{P}$ be the $p \times p$ population correlation matrix for $\mathbf{x}$ :

$$
\mathbf{P}=\boldsymbol{\Sigma}_{\mathrm{D}}^{-1 / 2} \boldsymbol{\Sigma} \boldsymbol{\Sigma}_{\mathrm{D}}^{-1 / 2}, \boldsymbol{\Sigma}_{\mathrm{D}}^{1 / 2}=\{\operatorname{Diag}(\boldsymbol{\Sigma})\}^{1 / 2}, \operatorname{Diag}(\mathbf{P})=\mathbf{I}_{p},
$$

where $\mathbf{I}_{p}$ is the $p \times p$ identity matrix. Then, $\lambda_{3}, \lambda_{2}$ and $\lambda_{6}$ with fixed weights $\mathbf{w}=\left(w_{1}, \ldots, w_{p}\right)^{\prime}$ for standardized variables, denoted by $\eta_{3}, \eta_{2}$ and $\eta_{6}$ respectively, are defined by replacing $\boldsymbol{\Sigma}$ with $\operatorname{diag}(\mathbf{w}) \mathbf{P} \operatorname{diag}(\mathbf{w})$ in (1.4), (3.1) and (3.3), respectively, where $\operatorname{diag}(\mathbf{w})$ is the diagonal matrix with the diagonal elements $w_{1}, \ldots, w_{p}$. The stratified and between-set versions of $\eta_{3}, \eta_{2}$ and $\eta_{6}$ are similarly defined. For instance, the generic expression corresponding to (2.1) becomes 


$$
\eta_{\mathrm{S}}=1-\frac{\sum_{I=1}^{B} \rho_{I}^{2}\left(1-\eta_{(I)}\right)}{\rho^{2}},
$$

where $\rho_{I}^{2}=\mathbf{w}_{(I)}^{\prime} \mathbf{P}_{I I} \mathbf{w}_{(I)}(I=1, \ldots, B) ; \mathbf{w}=\left(\mathbf{w}_{(1)}^{\prime}, \ldots, \mathbf{w}_{(B)}^{\prime}\right)^{\prime} ; \mathbf{w}_{(I)}=\left(w_{K+1}, \ldots\right.$, $\left.w_{K+p_{I}}\right)^{\prime}$ with $K=\left(\sum_{M=1}^{I} p_{M}\right)-p_{I} ; \mathbf{P}_{I I}$ is the $I$-th diagonal block of $\mathbf{P} ; \rho^{2}=\mathbf{w}^{\prime} \mathbf{P} \mathbf{w}$; and $\eta_{(I)}\left(\eta_{3(I)}, \eta_{2(I)}\right.$ or $\left.\eta_{6(I)}\right)$ denotes the coefficient defined for the $I$-th set similarly to $\eta_{3}, \eta_{2}$ and $\eta_{6}$ for all sets of variables. In these expressions $\rho_{I}^{2}, \rho^{2}, \eta_{(I)}$ and $\eta_{\mathrm{S}}$ depend on $\mathbf{w}$. However, the notation including $\mathbf{w}$ is avoided for simplicity.

\section{Sampling behavior of the estimators}

Sections 4 and 5 are for the sampling properties of reliability estimators based on asymptotic expansion. Readers who are not interested in this topic can skip these sections.

The sample counterparts of $\lambda$ 's and $\eta$ 's with and without stratification including fixed weights for $\eta$ 's are given by replacing $\boldsymbol{\Sigma}$ in $\lambda$ 's and $\mathbf{P}$ in $\eta$ 's by $\mathbf{S}$ and the sample correlation matrix $\mathbf{R}$, respectively, which gives the estimators $\hat{\lambda}$ 's and $\hat{\eta}$ 's. Let $\theta$ be one of the coefficients with its estimator $\hat{\theta}$. Note that $\hat{\theta}$ is a function of $\mathbf{s}$ and is differentiable with respect to $\mathbf{s}$ required number of times in a neighborhood of $\theta$. Assume that the moments of observed variables up to a required order exist. Let $y=n^{1 / 2}(\hat{\theta}-\theta)$, where $n+1=N$ is the number of independent observations. Then, the following asymptotic cumulants of $y$ are available (see e.g., Ogasawara, 2006a):

$$
\begin{aligned}
& \kappa_{1}(y)=\mathrm{E}(y)=n^{-1 / 2} \beta_{1}+O\left(n^{-3 / 2}\right), \\
& \kappa_{2}(y)=\mathrm{E}\left[\{\mathrm{y}-\mathrm{E}(y)\}^{2}\right]=\beta_{2}+n^{-1} \Delta \beta_{2}+O\left(n^{-2}\right), \\
& \kappa_{3}(y)=\mathrm{E}\left[\{\mathrm{y}-\mathrm{E}(y)\}^{3}\right]=n^{-1 / 2} \beta_{3}+O\left(n^{-3 / 2}\right), \\
& \kappa_{4}(y)=\mathrm{E}\left[\{\mathrm{y}-\mathrm{E}(y)\}^{4}\right]-3\left\{\kappa_{2}(y)\right\}^{2}=n^{-1} \beta_{4}+O\left(n^{-2}\right),
\end{aligned}
$$

where $n^{-1 / 2} \beta_{1}$ is the asymptotic bias of $y ; \beta_{2}$ is the asymptotic variance; $n^{-1} \Delta \beta_{2}$ is the higher-order (added) variance; $n^{-1 / 2} \beta_{3}$ is the asymptotic third cumulant or scaled skewness; and $n^{-1} \beta_{4}$ is the asymptotic fourth cumulant or scaled kurtosis. In (4.1), $\beta_{1}$ and $\beta_{2}$ are

$$
\beta_{1}=\frac{1}{2} \operatorname{tr}\left(\left.\frac{\partial^{2} \hat{\theta}}{\partial \mathbf{s} \partial \mathbf{s}^{\prime}}\right|_{\mathbf{s}=\boldsymbol{\sigma}} \boldsymbol{\Omega}\right), \beta_{2}=\left.\left.\frac{\partial \hat{\theta}}{\partial \mathbf{s}^{\prime}}\right|_{\mathbf{s}=\boldsymbol{\sigma}} \boldsymbol{\Omega} \frac{\partial \hat{\theta}}{\partial \mathbf{s}}\right|_{\mathbf{s}=\boldsymbol{\sigma}},
$$

where $(\boldsymbol{\Omega})_{a b, c d}=\sigma_{a b c d}-\sigma_{a b} \sigma_{c d}(p \geq a \geq b \geq 1 ; p \geq c \geq d \geq 1) ; n^{-1} \boldsymbol{\Omega}$ is the asymptotic covariance matrix of $\mathbf{s}$; and $(\cdot)_{a b, c d}$ denotes the element corresponding to the row of $\sigma_{a b}$ and the column of $\sigma_{c d}$; and $\sigma_{a b c d}$ is the fourth-order multivariate central moment of the variables $X_{a}, X_{b}, X_{c}$ and $X_{d}$. The expressions for $\Delta \beta_{2}, \beta_{3}$ and $\beta_{4}$ are available (see Ogasawara, 2006a), which include the moments up to the sixth order for $\Delta \beta_{2}$ and $\beta_{3}$, and up to the eighth order for $\beta_{4}$, and the partial derivatives of $\hat{\theta}$ with respect to $\mathrm{s}$ up to the second order for $\beta_{3}$ and up to the third order for $\Delta \beta_{2}$ and $\beta_{4}$, which are involved and not repeated here. When required moments of observed variables of $\hat{\theta}$ are available, the main task to obtain $\beta_{1}$ to $\beta_{4}$ is to derive the partial derivatives of $\hat{\theta}$ with respect to $\mathbf{s}$ up to the third order, which will be given in Appendix 1 . 
It is known that the asymptotic distribution function of $\hat{\theta}$ is given by the Edgeworth expansion as:

$$
\begin{aligned}
\operatorname{Pr}\left(\frac{y}{\beta_{2}^{1 / 2}} \leq z\right)= & \Phi(z)-n^{-1 / 2}\left\{\frac{\beta_{1}}{\beta_{2}^{1 / 2}}+\frac{\beta_{3}}{6 \beta_{2}^{3 / 2}}\left(z^{2}-1\right)\right\} \phi(z)-n^{-1}\left\{\frac{1}{2}\left(\Delta \beta_{2}+\beta_{1}^{2}\right) \frac{z}{\beta_{2}}\right. \\
& \left.+\left(\frac{\beta_{4}}{24}+\frac{\beta_{1} \beta_{3}}{6}\right) \frac{z^{3}-3 z}{\beta_{2}^{2}}+\frac{\beta_{3}^{2}\left(z^{5}-10 z^{3}+15 z\right)}{72 \beta_{2}^{3}}\right\} \phi(z)+o\left(n^{-1}\right),
\end{aligned}
$$

where $\phi(z)=(1 / \sqrt{2 \pi}) \exp \left(-z^{2} / 2\right)$ and $\Phi(z)=\int_{-\infty}^{z} \phi\left(z^{*}\right) d z^{*}$.

In (4.3) $\Phi(z)$ gives the usual normal approximation up to $O(1)$ using only $\beta_{2}$; and the expressions up to orders $O\left(n^{-1 / 2}\right)$ and $O\left(n^{-1}\right)$ are the single- and two-term Edgeworth expansions, respectively. It is known that the Edgeworth expanded distribution functions can be locally decreasing. These anomalous phenomena happen in the tail area. Hall's (1992) method of asymptotic expansion using variable transformation to remove asymptotic skewness does not give the decreasing distribution function:

$$
\begin{aligned}
& \operatorname{Pr}\left(\frac{y}{\beta_{2}^{1 / 2}} \leq z\right)=\Phi\{g(z)\}+o\left(n^{-1 / 2}\right), \\
& g(z)=\frac{2 n^{1 / 2} \beta_{2}^{3 / 2}}{\beta_{3}}\left[\left\{\frac{n^{-1 / 2} \beta_{3}}{6 \beta_{2}^{3 / 2}}\left(z-\frac{n^{-1 / 2} \beta_{1}}{\beta_{2}^{1 / 2}}\right)-1\right\}^{3}-1\right] .
\end{aligned}
$$

Equations (4.3) and (4.4) include asymptotic cumulants evaluated at the population values, which are usually unavailable in practice. Instead, the following Studentized statistic is available:

$$
t=\frac{n^{1 / 2}(\hat{\theta}-\theta)}{\hat{\beta}_{2}^{1 / 2}}=\frac{y}{\hat{\beta}_{2}^{1 / 2}},
$$

whose asymptotic cumulants are

$$
\kappa_{1}(t)=n^{-1 / 2} \beta_{1}^{\prime}+o\left(n^{-1 / 2}\right), \quad \kappa_{2}(t)=1+o(1), \quad \kappa_{3}(t)=n^{-1 / 2} \beta_{3}^{\prime}+o\left(n^{-1 / 2}\right),
$$

where $\beta_{1}^{\prime}$ and $\beta_{3}^{\prime}$ are given by Ogasawara (2007b) as

$$
\begin{aligned}
& \beta_{1}^{\prime}=\beta_{2}^{-1 / 2} \beta_{1}-\frac{1}{2} \beta_{2}^{-3 / 2}\left\{\left.\left.\frac{\partial \hat{\theta}}{\partial \mathbf{s}^{\prime}}\right|_{\mathbf{s}=\boldsymbol{\sigma}} \boldsymbol{\Omega} \frac{\partial \hat{\beta}_{2}}{\partial \mathbf{s}}\right|_{\mathbf{s}=\boldsymbol{\sigma}}+\left.\left.\frac{\partial \hat{\theta}}{\partial \mathbf{s}^{\prime}}\right|_{\mathbf{s}=\boldsymbol{\sigma}} n \operatorname{acov}\left(\mathbf{s}, \mathbf{s}_{(4)}^{\prime}\right) \frac{\partial \hat{\beta}_{2}}{\partial \mathbf{s}_{(4)}}\right|_{\mathbf{s}_{(4)}=\boldsymbol{\sigma}_{(4)}}\right\}, \\
& \beta_{3}^{\prime}=\beta_{2}^{-3 / 2} \beta_{3}-3 \beta_{2}^{-3 / 2}\left\{\left.\left.\frac{\partial \hat{\theta}}{\partial \mathbf{s}^{\prime}}\right|_{\mathbf{s}=\boldsymbol{\sigma}} \boldsymbol{\Omega} \frac{\partial \hat{\beta}_{2}}{\partial \mathbf{s}}\right|_{\mathbf{s}=\boldsymbol{\sigma}}+\left.\left.\frac{\partial \hat{\theta}}{\partial \mathbf{s}^{\prime}}\right|_{\mathbf{s}=\boldsymbol{\sigma}} n \operatorname{acov}\left(\mathbf{s}, \mathbf{s}_{(4)}^{\prime}\right) \frac{\partial \hat{\beta}_{2}}{\partial \mathbf{s}_{(4)}}\right|_{\mathbf{s}_{(4)}=\boldsymbol{\sigma}_{(4)}}\right\} .
\end{aligned}
$$

In (4.7) $\operatorname{acov}\left(\mathbf{s}, \mathbf{s}_{(4)}^{\prime}\right)$ is the asymptotic cross covariance matrix of $\mathbf{s}$ and $\mathbf{s}_{(4)}^{\prime}$, and $\mathbf{s}_{(4)}$ is the ${ }_{p+3} C_{4} \times 1$ vector of the nonduplicated sample fourth-order central moments with $\boldsymbol{\sigma}_{(4)}$ being its population counterpart.

The usual confidence interval with asymptotic confidence coefficient $1-\tilde{\alpha}$ (e.g., $\tilde{\alpha}=.05$ ) using the normal approximation is given by 


$$
\hat{\theta} \pm z_{\tilde{\alpha} / 2} n^{-1 / 2} \hat{\beta}_{2}^{1 / 2}
$$

where $z_{\tilde{\alpha} / 2}=\Phi^{-1}\{1-(\tilde{\alpha} / 2)\}$. The corresponding confidence interval using the CornishFisher expansion accurate up to order $O\left(n^{-1 / 2}\right)$ is

$$
\hat{\theta}+\left[ \pm z_{\tilde{\alpha} / 2}-n^{-1 / 2}\left\{\hat{\beta}_{1}^{\prime}+\left(\hat{\beta}_{3}^{\prime} / 6\right)\left(z_{\tilde{\alpha} / 2}^{2}-1\right)\right\}\right] n^{-1 / 2} \hat{\beta}_{2}^{1 / 2} .
$$

Hall's method gives the confidence interval with the same asymptotic accuracy as (4.9):

$$
\begin{aligned}
& \hat{\theta}-n^{-1} \hat{\beta}_{2}^{1 / 2} \hat{\beta}_{1}^{\prime} \\
& +6 \hat{\beta}_{2}^{1 / 2}\left(\hat{\beta}_{3}^{\prime}\right)^{-1}\left[\left\{1-(1 / 2) \hat{\beta}_{3}^{\prime}\left( \pm n^{-1 / 2} z_{\tilde{\alpha} / 2}-\left(n^{-1} / 6\right) \hat{\beta}_{3}^{\prime}\right)\right\}^{1 / 3}-1\right] .
\end{aligned}
$$

The above results were given under nonnormality. When normality holds, the results become considerably simple. The results associated with the cumulants of observed variables higher than 2 can be omitted. The first three asymptotic cumulants of the NT Studentized statistic under nonnormality, denoted by $\beta_{\mathrm{NT} 1}^{\prime \prime}, \beta_{\mathrm{NT} 2}^{\prime \prime}$ and $\beta_{\mathrm{NT} 3}^{\prime \prime}$ are also available (Ogasawara, 2007b):

$$
\begin{aligned}
& \beta_{\mathrm{NT} 1}^{\prime \prime}=\beta_{\mathrm{NT} 2}^{-1 / 2} \beta_{1}-\left.\left.\frac{1}{2} \beta_{\mathrm{NT} 2}^{-3 / 2} \frac{\partial \hat{\theta}}{\partial \mathbf{s}^{\prime}}\right|_{\mathbf{s}=\boldsymbol{\sigma}} \Omega \frac{\partial \hat{\beta}_{\mathrm{NT} 2}}{\partial \mathbf{s}}\right|_{\mathbf{s}=\boldsymbol{\sigma}}, \quad \beta_{\mathrm{NT} 2}^{\prime \prime}=\beta_{\mathrm{NT} 2}^{-1} \beta_{2}, \\
& \beta_{\mathrm{NT} 3}^{\prime \prime}=\beta_{\mathrm{NT} 2}^{-3 / 2} \beta_{3}-\left.\left.3 \beta_{\mathrm{NT} 2}^{-5 / 2} \beta_{2} \frac{\partial \hat{\theta}}{\partial \mathbf{s}^{\prime}}\right|_{\mathbf{s}=\boldsymbol{\sigma}} \Omega \frac{\partial \hat{\beta}_{\mathrm{NT} 2}}{\partial \mathbf{s}}\right|_{\mathbf{s}=\boldsymbol{\sigma}},
\end{aligned}
$$

where $\beta_{\mathrm{NT} 2}$ is $\beta_{2}$ under normality $\left(\beta_{\mathrm{NT} 1}^{\prime}, \beta_{\mathrm{NT} 2}^{\prime}\right.$ and $\beta_{\mathrm{NT} 3}^{\prime}$ are retained for the NT Studentized statistic under normality).

\section{The cases of equal asymptotic distributions for $\hat{\lambda}$ and $\hat{\eta}$}

The exact distributions of $\hat{\lambda}$ 's with unit population variances of observed variables are generally different from the corresponding distributions of $\hat{\eta}$ 's with unit weights. However, the asymptotic distributions become equal in some cases. For the derivation, assume that $\lambda$ and $\eta$ are unchanged by simultaneous multiplication of a common constant to the unstandardized variables or the weights for standardized variables, which is satisfied by the seven coefficients given earlier and is usually required for other coefficients. Then, the following theorem is presented.

Theorem 3. The asymptotic distribution of $\hat{\lambda}$ given by the normal approximation with equal population variances over observed variables and possibly with stratification is the same as that of $\hat{\eta}$ corresponding to $\hat{\lambda}$ with equal weights for standardized variables when

$$
\eta=\left\{\left.\frac{\partial h(\mathbf{r})}{\partial \mathbf{r}_{\cdot i}^{\prime}}\right|_{\mathbf{r}=\boldsymbol{\rho}} \boldsymbol{\rho}_{\cdot i}+\left.\frac{\partial h(\mathbf{r})}{\partial r_{i i}}\right|_{\mathbf{r}=\boldsymbol{\rho}}\right\} /\left(2 \mathbf{1}_{p}^{\prime} \boldsymbol{\rho}_{\cdot i}\right) \quad(i=1, \ldots, p),
$$

where $h(\boldsymbol{\rho})=\eta \rho^{2} ; \boldsymbol{\rho}=\mathrm{v}(\mathbf{P}) ; \mathbf{r}=\mathrm{v}(\mathbf{R}) ; \mathbf{r}_{\cdot i}$ is the $i$-th column of $\mathbf{R} ; \boldsymbol{\rho}_{\cdot i}$ is the $i$-th column of $\mathbf{P} ; r_{i i}$ in $h(\mathbf{r})$ is seen as a mathematical variable though actually $r_{i i}=\rho_{i i}=$ $1(i=1, \ldots, p)$.

The proof will be given in Appendix 2. 
Using Theorem 3, the following results are available.

Corollary 1. Under the conditions of Theorem 3, the asymptotic distribution of $\hat{\lambda}\left(\hat{\lambda}_{3}, \hat{\lambda}_{3 \mathrm{~S}}, \hat{\lambda}_{3 \mathrm{~T}}, \hat{\lambda}_{2}, \hat{\lambda}_{2 \mathrm{~S}}, \hat{\lambda}_{6}\right.$ or $\left.\hat{\lambda}_{6 \mathrm{~T}}\right)$ and that of the corresponding $\hat{\eta}$ are the same when $\mathbf{P}$ is compound symmetric for $B=1$, and $\mathbf{P}=\mathbf{I}_{B} \otimes \mathbf{P}_{1}+\rho_{0}\left(\mathbf{1}_{B} \mathbf{1}_{B}^{\prime}-\mathbf{I}_{B}\right) \otimes\left(\mathbf{1}_{p *} \mathbf{1}_{p *}^{\prime}\right)$ for $B>1$ with $p_{1}=\cdots=p_{B}=p^{*}$, where $\mathbf{P}_{1}$ is a $p^{*} \times p^{*}$ correlation matrix with compound symmetry and $\otimes$ denotes the Kronecker product.

Proof. Note that $\mathbf{P}$ for $B>1$ has the same within-set correlation matrices with compound symmetry, and has the equal off-block-diagonal elements $\rho_{0}$. Under the patterns of $\mathbf{P}$ with $B \geq 1$, the columns are the same except for the order of the elements in each column. Considering the symmetric properties of $\eta$ 's with respect to the order of variables and the patterns of $\mathbf{P}, \partial h(\mathbf{r}) /\left.\partial \mathbf{r}_{.}^{\prime}\right|_{\mathbf{r}=\boldsymbol{\rho}} \boldsymbol{\rho}_{{ }_{i}}$ and $\partial h(\mathbf{r}) /\left.\partial r_{i}\right|_{\mathbf{r}=\boldsymbol{\rho}}$ are unchanged over $i=1, \ldots, p$. The value of $\mathbf{1}_{p}^{\prime} \boldsymbol{\rho}_{\text {}_{i}}$ is the sum of the elements of $\boldsymbol{\rho}_{._{i}}$, which is also unchanged under the conditions. The equivalence of the common value of the right-hand sides of (5.1) over $i$ to $\eta$ is shown as follows. Taking the sum of the numerators and that of the denominators separately on the right-hand side, and having the ratio, (5.1) gives $\eta=\left\{\partial h(\boldsymbol{\rho}) / \partial \boldsymbol{\rho}^{\prime}\right\} \boldsymbol{\rho} / \rho$. This holds when $h(\boldsymbol{\rho})=\left\{\partial h(\boldsymbol{\rho}) / \partial \boldsymbol{\rho}^{\prime}\right\} \boldsymbol{\rho}$, which is satisfied by coefficients $\lambda_{3}$ through $\lambda_{6 \mathrm{~T}}$. Consequently, the equalities of (5.1) follow. Q. E. D.

Examples of $\mathbf{P}$ satisfying the condition of Theorem 3 for $B=1$ other than compound symmetry are found in Guttman's (1954) circumplex structure and its variations (see Browne, 1992).

\section{Numerical examples}

In this section, three artificial examples are presented for illustration and confirmation of the asymptotic results in finite samples. The first two examples are based on fourvariable covariance matrices and the third example uses a six-variable covariance matrix. They are all positive definite with unit population variances:

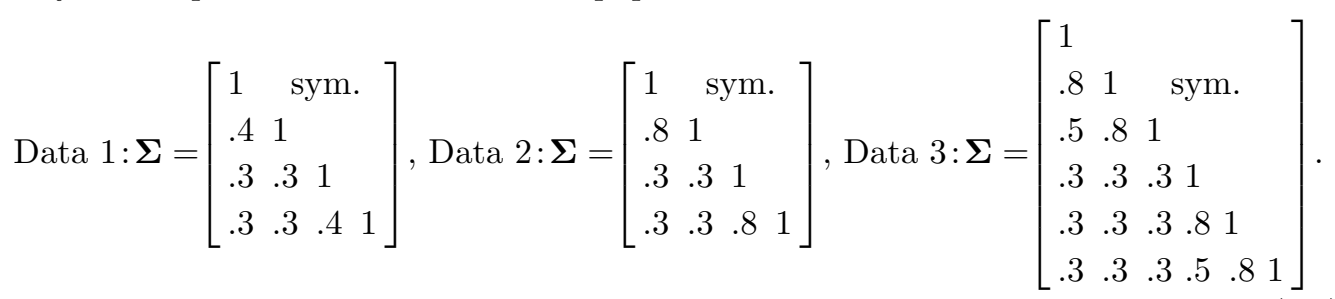

When stratified/between-set coefficients are used, the first and second halves of the variables in each example are seen as strata or sets of components $(B=2)$. With stratification, the within-set covariances in Data 1-3 are relatively higher than the between-set covariances. Data 2 has a within-set covariance higher than that of Data 1 . Note that each of Data 1 and 2 has the same columns except for the order of the elements in each column while Data 3 does not have this property.

Simulations were performed using the population covariance matrices in (6.1) under normality and nonnormality. Random observations were generated by $\mathbf{x}=\mathbf{A f}$ with $\boldsymbol{\Sigma}=\mathbf{A} \mathbf{A}^{\prime}$, where $\mathbf{A}$ is the Cholesky-decomposed lower-triangular matrix of $\boldsymbol{\Sigma}, \mathbf{f}$ is the $p \times 1$ random 
Table 1: Simulated and theoretical $\sqrt{n}$ times standard errors $\left(\beta_{2}^{1 / 2}=\sqrt{n}\right.$ ASE $)$ of the nonStudentized estimators for four-variable data sets $(n=50)$

\begin{tabular}{|c|c|c|c|c|c|c|c|c|c|c|}
\hline & \multicolumn{5}{|c|}{ Data 1 (Low within-set correlation) } & \multicolumn{5}{|c|}{ Data 2 (High within-set correlation) } \\
\hline & \multirow[b]{2}{*}{ Coef. } & \multicolumn{2}{|c|}{ Unstandardized } & \multicolumn{2}{|c|}{ Standardized } & \multirow[b]{2}{*}{ Coef. } & \multicolumn{2}{|c|}{ Unstandardized } & \multicolumn{2}{|c|}{ Standardized } \\
\hline & & Normal & $\mathrm{C} 1$ & Normal & $\mathrm{C} 1$ & & Normal & $\mathrm{C} 1$ & Normal & $\mathrm{C} 1$ \\
\hline$\lambda_{3}, \eta_{3}$ & .667 & & & & & .778 & & & & \\
\hline Sim. & & .59 & .71 & .59 & .70 & & .43 & .50 & .43 & .51 \\
\hline Th. & & .55 & .71 & * & * & & .40 & .51 & * & $*$ \\
\hline$\lambda_{3 \mathrm{~S}}, \eta_{3 \mathrm{~S}}$ & .700 & & & & & .917 & & & & \\
\hline Sim. & & .56 & .69 & .56 & .68 & & .16 & .30 & .16 & .29 \\
\hline Th. & & .52 & .69 & * & * & & .14 & .28 & * & * \\
\hline$\lambda_{3 \mathrm{~T}}, \eta_{3 \mathrm{~T}}$ & .600 & & & & & .500 & & & & \\
\hline Sim. & & .86 & 1.02 & .86 & 1.01 & & 1.08 & 1.19 & 1.08 & 1.22 \\
\hline Th. & & .80 & 1.02 & * & * & & 1.00 & 1.24 & $*$ & * \\
\hline$\lambda_{2}, \eta_{2}$ & .668 & & & & & .801 & & & & \\
\hline Sim. & & .55 & .67 & .55 & .66 & & .29 & .38 & .30 & .39 \\
\hline Th. & & .54 & .71 & $*$ & $*$ & & .29 & .40 & $*$ & $*$ \\
\hline$\lambda_{2 \mathrm{~S}}, \eta_{2 \mathrm{~S}}$ & .700 & & & & & .917 & & & & \\
\hline Sim. & & .56 & .69 & .56 & .67 & & .16 & .30 & .16 & .29 \\
\hline Th. & & .52 & .69 & * & * & & .14 & .28 & * & * \\
\hline$\lambda_{6}, \eta_{6}$ & .607 & & & & & .852 & & & & \\
\hline Sim. & & .59 & .74 & .59 & .72 & & .25 & .43 & .25 & .42 \\
\hline Th. & & .58 & .77 & * & $*$ & & .24 & .45 & $*$ & $*$ \\
\hline$\lambda_{6 \mathrm{~T}}, \eta_{6 \mathrm{~T}}$ & .429 & & & & & .333 & & & & \\
\hline Sim. & & .81 & 1.00 & .81 & .99 & & .88 & 1.05 & .88 & 1.05 \\
\hline Th. & & .82 & 1.04 & * & $*$ & & .89 & 1.10 & * & $*$ \\
\hline
\end{tabular}

Note. $n+1=$ The sample size in the simulation, Coef. $=$ Population coefficient value, Normal $=$ Normally distributed data. $\mathrm{C} 1=$ Chi-square distributed data with 1 degree of freedom, Sim. $=$ Simulated value, Th. $=$ Theoretical or asymptotic value. The asterisks indicate that the corresponding values for unstandardized data hold.

vector with each element being independently distributed. Nonnormal data were given by the independently chi-square distributed variable with 1 degree of freedom and standardization to have unit variance in each element of $\mathbf{f}$. The sample size $n=N-1=50$ or 200 is used in the simulations. From a set of generated observations, $\mathbf{S}$ and $\mathbf{R}$ were given, which yielded $\hat{\lambda}$ 's and $\hat{\eta}$ 's. This procedure was replicated 1,000,000 times. From the 1,000,000 estimates of each coefficient, the sample cumulants were given by the $k$-statistics (unbiased estimators of cumulants; Stuart \& Ort, 1994, Equation (12.28)) multiplied by appropriate powers of $n$ for ease of comparison to the corresponding asymptotic cumulants. The higher-order asymptotic standard error (HASE) is defined as $\sqrt{\left(\beta_{2} / n\right)+\left(\Delta \beta_{2} / n^{2}\right)}$ whose sample counterpart is the simulated standard error $(\mathrm{SE})$, which is the usual standard deviation (SD) given by the square root of an unbiased sample variance for a coefficient based on 1,000,000 estimates while the usual asymptotic standard error ASE $=\sqrt{\beta_{2} / n}$. NT Studentized estimates were also constructed using sample NT ASEs under normality and nonnormality.

Table 1 shows the theoretical and simulated SEs as well as the population values of the 
Table 2: Simulated and theoretical cumulants of the non-Studentized estimators for six-variable unstandardized data (Data 3)

\begin{tabular}{lrcrrrrrrr}
\hline \multicolumn{2}{c}{ Coefficient } & \multicolumn{2}{c}{$\beta_{2}^{1 / 2}$ (dispersion) } & \multicolumn{2}{c}{$\beta_{1}$ (bias) } & \multicolumn{2}{c}{$\beta_{3}$ (skewness) } & \multicolumn{2}{c}{$\beta_{4}$ (kurtosis) } \\
& \multicolumn{1}{c}{$n$} & Normal & \multicolumn{1}{c}{ C1 } & Normal & \multicolumn{1}{c}{ C1 } & Normal & C1 & Normal & C1 \\
\hline$\lambda_{3}$ & 50 & .29 & .35 & -.34 & -.55 & -.16 & -.20 & .6 & .5 \\
.836 & 200 & .28 & .35 & -.33 & -.58 & -.13 & -.19 & .4 & .5 \\
& Th. & .27 & .35 & -.33 & -.61 & -.12 & -.18 & .3 & .5 \\
\hline$\lambda_{3 \mathrm{~S}}$ & 50 & .16 & .22 & -.19 & -.37 & -.03 & -.06 & .1 & .1 \\
.909 & 200 & .15 & .22 & -.18 & -.38 & -.02 & -.06 & .0 & .1 \\
& Th. & .15 & .22 & -.18 & -.40 & -.02 & -.05 & .0 & .1 \\
\hline$\lambda_{3 \mathrm{~T}}$ & 50 & .98 & 1.10 & -.95 & -1.27 & -6.01 & -6.78 & 72.5 & 51.3 \\
.545 & 200 & .93 & 1.11 & -.93 & -1.36 & -4.87 & -7.25 & 51.4 & 61.7 \\
& Th. & .91 & 1.12 & -.91 & -1.44 & -4.51 & -7.42 & 45.1 & 74.3 \\
\hline$\lambda_{2}$ & 50 & .22 & .27 & -.04 & -.01 & -.05 & -.06 & .1 & .1 \\
.851 & 200 & .22 & .28 & -.04 & .01 & -.04 & -.05 & .1 & .1 \\
& Th. & .22 & .29 & -.04 & .01 & -.04 & -.04 & .1 & .1 \\
\hline$\lambda_{2 \mathrm{~S}}$ & 50 & .15 & .19 & -.12 & -.16 & -.02 & -.04 & .0 & .1 \\
.913 & 200 & .14 & .20 & -.12 & -.16 & -.02 & -.04 & .0 & .1 \\
& Th. & .14 & .20 & -.12 & -.17 & -.01 & -.04 & .0 & .1 \\
\hline$\lambda_{6}$ & 50 & .12 & .18 & .23 & .30 & -.01 & -.04 & .0 & .1 \\
.926 & 200 & .12 & .19 & .23 & .32 & -.01 & -.04 & .0 & .1 \\
& Th. & .12 & .20 & .22 & .33 & -.01 & -.04 & .0 & .1 \\
\hline$\lambda_{6 \mathrm{~T}}$ & 50 & .83 & 1.00 & 1.03 & 1.28 & -1.30 & -1.02 & 2.1 & -10.1 \\
.384 & 200 & .85 & 1.04 & 1.00 & 1.30 & -1.41 & -.76 & 2.4 & -9.6 \\
& Th. & .85 & 1.05 & .99 & 1.29 & -1.44 & -.51 & 2.6 & -3.4 \\
\hline
\end{tabular}

Note. $n+1=$ The sample size in the simulation. Th. = Theoretical or asymptotic value, Normal $=$ Normally distributed data, $\mathrm{C} 1=$ Chi-square distributed data with 1 degree of freedom.

coefficients for Data 1 and 2 with $\mathbf{w}=\mathbf{1}_{p}$ for $\eta$ 's (i.e., $\lambda=\eta$ ). The effect of stratification is considerable. $\lambda_{3 \mathrm{~S}}\left(\lambda_{2 \mathrm{~S}}\right)$ in Data 2 is as high as .917 while $\lambda_{3 \mathrm{~T}}=.500$ and $\lambda_{6 \mathrm{~T}}=.333$. However, it is to be noted that $\lambda_{3 \mathrm{~S}}\left(\lambda_{2 \mathrm{~S}}\right)$ is not necessarily better than $\lambda_{3 \mathrm{~T}}$ and $\lambda_{6 \mathrm{~T}}$ in this case since, as addressed earlier, the high-within set covariance/correlation may be due to the errors highly correlated within each set. On the other hand, in many practical cases, the high correlations can be considered as those due to part of true scores. In both extreme cases, $\lambda_{3}$ and $\lambda_{2}$ with intermediate values do not represent such structures. In the table $\lambda_{3 \mathrm{~S}}=\lambda_{2 \mathrm{~S}}$ with their ASEs being equal. Though not shown in the table, the remaining corresponding asymptotic cumulants are also equal. This property stems from $\hat{\lambda}_{3 \mathrm{~S}}=\hat{\lambda}_{2 \mathrm{~S}}$ which holds when the numbers of within-set components are all two and the within-set cross covariances are nonnegative. This is easily found from the sample counterpart of (3.2), where $\left[\left\{p_{I} /\left(p_{I}-1\right)\right\} \sum_{i \neq j} s_{I_{i} I_{j}}^{2}\right]^{1 / 2}$ for $\hat{\lambda}_{2 \mathrm{~S}}$ is the sum of reproduced true-score sample variances, which becomes $2\left|s_{I_{i} I_{j}}\right|$ whose counterpart for $\hat{\lambda}_{3 \mathrm{~S}}$ is $2 s_{I_{i} I_{j}}$ (see (2.2)). The asterisks in the table show the equality of the results for unstandardized variables and standardized variables given in Corollary 1. The simulated/asymptotic SEs under nonnormality are larger than those under normality, which is explained by the large kurtosis of the chi-square distribution with 1 degree of freedom. In the table it is found 
Table 3: Simulated and theoretical SE ratios of the non-Studentized estimators and cumulants of the Studentized estimators in six-variable unstandardized data (Data 3)

\begin{tabular}{|c|c|c|c|c|c|c|c|c|c|}
\hline & $n$ & $\mathrm{SD} / \mathrm{ASE}$ & $\begin{array}{l}\text { HASE/ASE } \\
\mathrm{C} 1\end{array}$ & $\begin{array}{c}\beta_{\mathrm{NT} 2}^{1 / 2^{\prime}} \\
\text { Normal } \\
\end{array}$ & $\begin{array}{c}\beta_{\mathrm{NT} 2}^{1 / 2}{ }^{\prime \prime} \\
\mathrm{C} 1\end{array}$ & $\begin{array}{c}\beta_{\mathrm{NT} 1}^{\prime} \\
\text { Normal }\end{array}$ & $\begin{array}{c}\beta_{\mathrm{NT} 1}^{\prime \prime} \\
\mathrm{C} 1 \\
\end{array}$ & $\begin{array}{c}\beta_{\mathrm{NT} 3}^{\prime} \\
\text { Normal } \\
\end{array}$ & $\begin{array}{c}\beta_{\mathrm{NT3}}^{\prime \prime} \\
\mathrm{C} 1 \\
\end{array}$ \\
\hline$\lambda_{3}$ & $\begin{array}{r}50 \\
200 \\
\text { Th. }\end{array}$ & $\begin{array}{l}.998 \\
.993\end{array}$ & $\begin{array}{l}.955 \\
.989\end{array}$ & $\begin{array}{l}1.04 \\
1.01 \\
1\end{array}$ & $\begin{array}{l}1.36 \\
1.31 \\
1.29\end{array}$ & $\begin{array}{l}.50 \\
.49 \\
.50\end{array}$ & $\begin{array}{l}.59 \\
.59 \\
.55\end{array}$ & $\begin{array}{l}5.0 \\
4.6 \\
4.4\end{array}$ & $\begin{array}{l}22.3 \\
19.6 \\
18.8\end{array}$ \\
\hline$\lambda_{3 \mathrm{~S}}$ & $\begin{array}{r}50 \\
200 \\
\text { Th. }\end{array}$ & $\begin{array}{l}1.026 \\
1.001\end{array}$ & $\begin{array}{l}.990 \\
.998\end{array}$ & $\begin{array}{l}1.03 \\
1.01 \\
1\end{array}$ & $\begin{array}{l}1.51 \\
1.46 \\
1.44\end{array}$ & $\begin{array}{l}.45 \\
.45 \\
.45\end{array}$ & $\begin{array}{l}.76 \\
.77 \\
.74\end{array}$ & $\begin{array}{l}4.8 \\
4.4 \\
4.2\end{array}$ & $\begin{array}{l}30.8 \\
27.4 \\
26.3\end{array}$ \\
\hline$\lambda_{3 \mathrm{~T}}$ & $\begin{array}{r}50 \\
200 \\
\text { Th. }\end{array}$ & $\begin{array}{l}.990 \\
.994\end{array}$ & $\begin{array}{l}.960 \\
.990\end{array}$ & $\begin{array}{l}1.09 \\
1.02 \\
1\end{array}$ & $\begin{array}{l}1.38 \\
1.26 \\
1.23\end{array}$ & $\begin{array}{l}1.05 \\
1.00 \\
1.00\end{array}$ & $\begin{array}{l}1.46 \\
1.45 \\
1.43\end{array}$ & $\begin{array}{l}8.5 \\
6.5 \\
6.0\end{array}$ & $\begin{array}{l}27.3 \\
19.4 \\
17.3\end{array}$ \\
\hline$\lambda_{2}$ & $\begin{array}{r}50 \\
200 \\
\text { Th. }\end{array}$ & $\begin{array}{l}.932 \\
.975\end{array}$ & $\begin{array}{l}.869 \\
.969\end{array}$ & $\begin{array}{l}1.05 \\
1.01 \\
1\end{array}$ & $\begin{array}{l}1.45 \\
1.35 \\
1.32\end{array}$ & $\begin{array}{l}.98 \\
.96 \\
.96\end{array}$ & $\begin{array}{l}2.04 \\
2.10 \\
2.10\end{array}$ & $\begin{array}{l}3.3 \\
2.8 \\
2.7\end{array}$ & $\begin{array}{l}26.2 \\
20.0 \\
18.0\end{array}$ \\
\hline$\lambda_{2 \mathrm{~S}}$ & $\begin{array}{r}50 \\
200 \\
\text { Th. }\end{array}$ & $\begin{array}{l}.971 \\
.984\end{array}$ & $\begin{array}{l}.914 \\
.979\end{array}$ & $\begin{array}{l}1.04 \\
1.01 \\
1\end{array}$ & $\begin{array}{l}1.57 \\
1.48 \\
1.45\end{array}$ & $\begin{array}{l}.73 \\
.72 \\
.73\end{array}$ & $\begin{array}{l}1.90 \\
1.98 \\
2.00\end{array}$ & $\begin{array}{l}4.5 \\
4.1 \\
3.9\end{array}$ & $\begin{array}{l}36.2 \\
29.3 \\
27.1\end{array}$ \\
\hline$\lambda_{6}$ & $\begin{array}{r}50 \\
200 \\
\text { Th. }\end{array}$ & $\begin{array}{l}.914 \\
.970\end{array}$ & $\begin{array}{l}.846 \\
.964\end{array}$ & $\begin{array}{l}1.15 \\
1.03 \\
1\end{array}$ & $\begin{array}{l}2.14 \\
1.76 \\
1.65\end{array}$ & $\begin{array}{l}3.87 \\
3.56 \\
3.46\end{array}$ & $\begin{array}{l}7.51 \\
7.07 \\
6.98\end{array}$ & $\begin{array}{l}6.3 \\
4.4 \\
3.9\end{array}$ & $\begin{array}{r}104.8 \\
53.2 \\
42.6\end{array}$ \\
\hline$\lambda_{6 \mathrm{~T}}$ & $\begin{array}{r}50 \\
200 \\
\text { Th. }\end{array}$ & $\begin{array}{l}.950 \\
.986\end{array}$ & $\begin{array}{l}.973 \\
.993\end{array}$ & $\begin{array}{l}1.10 \\
1.02 \\
1\end{array}$ & $\begin{array}{l}1.47 \\
1.29 \\
1.24\end{array}$ & $\begin{array}{l}2.16 \\
1.99 \\
1.94\end{array}$ & $\begin{array}{l}3.05 \\
2.80 \\
2.70\end{array}$ & $\begin{array}{l}4.1 \\
2.6 \\
2.3\end{array}$ & $\begin{array}{l}22.4 \\
12.1 \\
10.1\end{array}$ \\
\hline
\end{tabular}

Note. $n+1=$ The sample size in the simulation and the theoretical ratio (HASE/ $\mathrm{ASE}) . \mathrm{SD}=$ The standard deviation from the simulation, $\mathrm{ASE}=\sqrt{\beta_{2} / n}, \mathrm{HASE}$ $=\sqrt{\left(\beta_{2} / n\right)+\left(\Delta \beta_{2} / n^{2}\right)}$, Normal $=$ Normally distributed data, $\mathrm{C} 1=$ Chi-square distributed data with 1 degree of freedom, Th. = Theoretical or asymptotic value.

that when the values of coefficients are high, their simulated/asymptotic SEs are small. It is known that the exact variance of $\hat{\lambda}_{3}$ under normality with compound symmetry is proportional to $\left(1-\lambda_{3}\right)^{2}$ (Kristof, 1963, Equations (58) and (63); see also Ogasawara, $2006 \mathrm{~b})$. This can be interpreted as a ceiling effect of $\lambda$ 's whose upper bound is 1 . The effect seems to be conveyed to the small SEs of $\hat{\lambda}_{3 \mathrm{~S}}, \hat{\lambda}_{2 \mathrm{~S}}, \hat{\eta}_{3 \mathrm{~S}}$ and $\hat{\eta}_{2 \mathrm{~S}}$ under different conditions.

Tables 2 and 3 show the results for unstandardized variables in Data 3, whose simulated values are given with $n=50$ and 200 similarly to those in Table 1 . In the tables, the results of the cumulants and HASE ratios (HASE/ASE and its sample counterpart SD/ASE) under normality/nonnormality are presented. The highest coefficient is $\lambda_{6}=.926$ followed by the second highest $\lambda_{2 \mathrm{~S}}=.913$ which is slightly higher than $\lambda_{3 \mathrm{~S}}=.909$. The lowest coefficient is $\lambda_{6 \mathrm{~T}}=.384$. The simulated values in the tables with $n=50$ and 200 similar to their asymptotic values, especially when $n=200$, indicate the usefulness of the asymptotic cumulants in finite samples. The absolute values of simulated/asymptotic third and fourth cumulants for $\hat{\lambda}_{3 \mathrm{~T}}$ and $\hat{\lambda}_{6 \mathrm{~T}}$ are relatively larger than those of other estimators 
Table 4: Theoretical cumulants of the non-Studentized and Studentized estimators, and HASE/ ASE of the non-Studentized estimators in six-variable standardized data (Data 3)

\begin{tabular}{cccccccrr}
\hline & \multicolumn{2}{c}{$\beta_{2}^{1 / 2}$ (dispersion) } & \multicolumn{2}{c}{$\beta_{1}$ (bias) } & \multicolumn{2}{c}{$\beta_{3}$ (skewness) } & \multicolumn{2}{c}{$\beta_{4}$ (kurtosis) } \\
Coefficient & Normal & C1 & Normal & C1 & Normal & C1 & Normal & C1 \\
\hline$\lambda_{3}$ & .27 & .35 & -.28 & -.37 & -.12 & -.16 & .3 & .5 \\
$\lambda_{3 \mathrm{~S}}$ & .15 & .21 & -.16 & -.30 & -.02 & -.05 & .0 & .1 \\
$\lambda_{3 \mathrm{~T}}$ & .91 & 1.11 & -.77 & -.63 & -4.51 & -6.18 & 45.1 & 51.6 \\
$\lambda_{2}$ & .22 & .28 & -.09 & -.11 & -.04 & -.05 & .1 & .1 \\
$\lambda_{2 \mathrm{~S}}$ & .13 & .20 & -.12 & -.22 & -.01 & -.03 & .0 & .1 \\
$\lambda_{6}$ & .12 & .19 & .18 & .15 & -.01 & -.04 & .0 & .1 \\
$\lambda_{6 \mathrm{~T}}$ & .85 & 1.05 & 1.04 & 1.68 & -1.44 & -.01 & 2.6 & -6.7 \\
\hline & HASE/ASE & $(n=50)$ & $\beta_{\text {NT2 }}^{1 / 2}$ & $\beta_{\text {NT2 }}^{1 / 2}$ & $\beta_{\text {NT1 }}^{\prime}$ & $\beta_{\text {NT1 }}^{\prime \prime}$ & $\beta_{\text {NT3 }}^{\prime}$ & $\beta_{\text {NT3 }}^{\prime \prime}$ \\
& Normal & C1 & Normal & C1 & Normal & C1 & Normal & C1 \\
\hline$\lambda_{3}$ & 1.075 & .964 & 1 & 1.29 & .66 & 1.44 & 4.4 & 19.7 \\
$\lambda_{3 \mathrm{~S}}$ & 1.075 & .975 & 1 & 1.45 & .50 & 1.31 & 4.0 & 27.4 \\
$\lambda_{3 \mathrm{~T}}$ & 1.070 & .959 & 1 & 1.23 & 1.15 & 2.31 & 6.0 & 18.9 \\
$\lambda_{2}$ & 1.021 & .913 & 1 & 1.32 & .73 & 1.52 & 2.6 & 16.0 \\
$\lambda_{2 \mathrm{~S}}$ & 1.061 & .938 & 1 & 1.47 & .55 & 1.52 & 3.6 & 26.5 \\
$\lambda_{6}$ & 1.008 & .849 & 1 & 1.65 & 3.04 & 5.45 & 3.8 & 41.4 \\
$\lambda_{6 \mathrm{~T}}$ & .986 & .936 & 1 & 1.24 & 2.00 & 3.16 & 2.3 & 10.9 \\
\hline
\end{tabular}

Note. $n+1=$ The sample size in HASE/ASE $=\sqrt{\left(\beta_{2} / n\right)+\left(\Delta \beta_{2} / n^{2}\right)} / \sqrt{\beta_{2} / n}$. Normal $=$ Normally distributed data, $\mathrm{C} 1=$ Chi-square distributed data with 1 degree of freedom.

though the formers do not give relatively large values when Studentized. The ratios of HASE/ASE with $n=50$ under nonnormality are somewhat more extreme than those with $n=200$ when compared to the corresponding simulated ratios (SD/ASE).

Table 4 shows the asymptotic results for standardized variables with unit weights in Data 3. The ASEs are not equal to the corresponding ones in Table 2 though they are mostly the same up to the second place.

The theoretical values in Table 1 to 4 were given by using population values of $\boldsymbol{\Sigma}(=\mathbf{P})$. In order to confirm the accuracy of interval estimation using sample cumulants, additional simulations were carried out under normality with $n=50$ for unstandardized variables in Data 3. Three confidence intervals were constructed using (4.9), (4.10) and (4.11) with the sample cumulants for the NT Studentized estimators under normality in each replication of the simulation. The number of replications was reduced to 100,000 to save computation time. Table 5 shows the proportions of true parameters below the lower endpoints of confidence intervals for selected three coefficients $\lambda_{3 S}, \lambda_{2}$ and $\lambda_{6}$. The table shows unsatisfactory results of the usual normal approximation while the other two methods give reasonable and similar results.

Table 6 gives the root mean square errors of the asymptotic approximations to the distribution functions of the standardized estimators $\left(\sqrt{n}(\hat{\theta}-\theta) / \beta_{2}^{1 / 2}\right)$ by the four asymptotic methods, where the mean of errors is given by averaging over the 40 points, $-3.8,-3.6, \ldots, 4.0$, and an error is defined as an asymptotic approximation minus the corresponding simulated proportion. Table 6 shows that the two-term Edgeworth expansion gives reduced errors especially when $n=200$ while the improvements of the single-term 
Table 5: Simulated proportions below the lower endpoints of the confidence intervals based on the normal-theory Studentized estimators under normality in six-variable unstandardized data $(n=50$; Data 3$)$

\begin{tabular}{lllllllll}
\hline \multicolumn{2}{c}{ Coefficient } & \multicolumn{7}{c}{ Nominal values } \\
& Method & .0050 & .0250 & .1000 & .5000 & .9000 & .9750 & .9950 \\
\hline$\lambda_{3 S}$ & $\mathrm{~N}^{*}$ & .0185 & .0466 & .1205 & .4850 & .9220 & .9926 & .9998 \\
& $\mathrm{C}-\mathrm{F}$ & .0062 & .0275 & .1005 & .4982 & .8930 & .9695 & .9915 \\
& Hall & .0027 & .0223 & .0988 & .4977 & .8948 & .9758 & .9959 \\
\hline$\lambda_{2}$ & $\mathrm{~N}^{*}$ & .0182 & .0505 & .1350 & .5296 & .9243 & .9860 & .9984 \\
& $\mathrm{C}-\mathrm{F}$ & .0061 & .0275 & .1054 & .5000 & .8927 & .9720 & .9938 \\
& Hall & .0035 & .0240 & .1045 & .5000 & .8930 & .9729 & .9943 \\
\hline$\lambda_{6}$ & $\mathrm{~N}^{*}$ & .0507 & .1127 & .2397 & .6601 & .9665 & .9973 & .9999 \\
& $\mathrm{C}-\mathrm{F}$ & .0118 & .0424 & .1313 & .5150 & .8810 & .9595 & .9869 \\
& Hall & .0065 & .0361 & .1299 & .5148 & .8824 & .9652 & .9920 \\
\hline
\end{tabular}

Note. $n+1=$ The sample size in the simulation. $\mathrm{N}^{*}=$ Normal approximation, $\mathrm{C}-\mathrm{F}=$ The

Cornish-Fisher expansion, Hall = Hall's (1992) method by variable transformation.

Table 6 : $10^{5} \times$ Root mean square errors of the asymptotic distribution functions of the standardized estimators in six-variable unstandardized data (Data 3)

\begin{tabular}{crrrrrrrr}
\hline & \multicolumn{4}{c}{ Normal } & \multicolumn{5}{c}{ Chi-square with 1 degree of freedom } \\
& $\mathrm{N}^{*}$ & $\mathrm{E} 1$ & $\mathrm{E} 2$ & Hall & \multicolumn{1}{c}{$\mathrm{N}^{*}$} & $\mathrm{E} 1$ & $\mathrm{E} 2$ & Hall \\
\hline$n=50$ & & & & & & & & \\
$\lambda_{3}$ & 2658 & 571 & 187 & 461 & 3525 & 775 & 745 & 694 \\
$\lambda_{3 \mathrm{~S}}$ & 2687 & 574 & 179 & 465 & 3742 & 865 & 737 & 682 \\
$\lambda_{3 \mathrm{~T}}$ & 2302 & 524 & 186 & 454 & 2631 & 804 & 683 & 725 \\
$\lambda_{2}$ & 1390 & 288 & 91 & 310 & 1477 & 1148 & 908 & 1153 \\
$\lambda_{2 \mathrm{~S}}$ & 1993 & 426 & 143 & 345 & 2095 & 1076 & 772 & 935 \\
$\lambda_{6}$ & 6673 & 804 & 211 & 888 & 6039 & 1169 & 1014 & 2098 \\
$\lambda_{6 \mathrm{~T}}$ & 3823 & 259 & 175 & 361 & 3528 & 437 & 371 & 584 \\
\hline$n=200$ & & & & & & & & \\
$\lambda_{3}$ & 1331 & 152 & 35 & 118 & 1862 & 231 & 147 & 211 \\
$\lambda_{3 \mathrm{~S}}$ & 1333 & 149 & 32 & 111 & 1971 & 251 & 130 & 189 \\
$\lambda_{3 \mathrm{~T}}$ & 1164 & 139 & 30 & 104 & 1360 & 257 & 118 & 218 \\
$\lambda_{2}$ & 700 & 71 & 27 & 71 & 556 & 379 & 149 & 381 \\
$\lambda_{2 \mathrm{~S}}$ & 997 & 112 & 27 & 78 & 990 & 359 & 112 & 328 \\
$\lambda_{6}$ & 3267 & 187 & 25 & 209 & 3036 & 350 & 148 & 596 \\
$\lambda_{6 \mathrm{~T}}$ & 1861 & 65 & 29 & 78 & 1729 & 125 & 108 & 170 \\
\hline
\end{tabular}

Note. $n+1=$ The sample size in the simulation. $\mathrm{N}^{*}=$ Normal approximation, $\mathrm{E} 1=$ The single-term Edgeworth expansion, E2 = The two-term Edgeworth expansion, Hall = Hall's (1992) method by variable transformation.

Edgeworth expansion and Hall's method over the normal approximation are similar.

\section{Discussion}

In the previous sections, stratified/between-set coefficients for reliability have been confined to those of $\lambda_{3}, \lambda_{2}$ and $\lambda_{6}$, and their standardized-variable versions. As addressed 
earlier, the formula of (2.1) for stratified coefficients is a general one, and can, in principle, be applied to other coefficients. It is known that the greatest lower bound $\lambda_{\text {glb }}$ of $\rho_{X X^{\prime}}$ for unstandardized variables is not less than other $\lambda$ 's (Bentler, 1972; Jackson \& Agunwanba, 1977; Woodhouse \& Jackson, 1977; Bentler \& Woodward, 1980; ten Berge, Snijders \& Zegers, 1981; ten Berge \& Socan, 2004). This corresponds to the problem of searching the nonnegative definite diagonal $\boldsymbol{\Psi}^{*}$ which maximizes $\operatorname{tr}\left(\boldsymbol{\Psi}^{*}\right)$, when given $\boldsymbol{\Sigma}$, with $\boldsymbol{\Sigma}-\boldsymbol{\Psi}^{*}$ being positive semidefinite. Stratified $\lambda_{\text {glb }}\left(\lambda_{\text {glb S }}\right)$ may be defined as:

$$
\lambda_{\mathrm{glbS}}=1-\left\{\sum_{I=1}^{B} \sigma_{I}^{2}\left(1-\lambda_{\mathrm{glb}(I)}\right) / \sigma^{2}\right\},
$$

where $\lambda_{\mathrm{glb}(I)}$ is given from the $I$-th diagonal block of $\boldsymbol{\Sigma}$ in a manner similar to $\lambda_{\mathrm{glb}}$. The between-set version of $\lambda_{\text {glb }}$ may also be defined as

$$
\lambda_{\mathrm{glb} \mathrm{T}}=\mathbf{1}_{p}^{\prime}\left(\boldsymbol{\Sigma}-\boldsymbol{\Psi}_{\mathrm{B}}^{*}\right) \mathbf{1}_{p} / \sigma^{2},
$$

where $\boldsymbol{\Psi}_{\mathrm{B}}^{*}$ takes a block diagonal form with nonnegative definite diagonal blocks and $\mathbf{1}_{p}^{\prime} \boldsymbol{\Psi}_{\mathrm{B}}^{*} \mathbf{1}_{p}$ is maximized on condition that $\boldsymbol{\Sigma}-\mathbf{\Psi}_{\mathrm{B}}^{*}$ is positive semidefinite. To the author's knowledge, this is a new problem with unknown sampling behavior of $\hat{\lambda}_{\text {glb T }}$ (for the asymptotic results of $\hat{\lambda}_{\text {glb }}$ see Shapiro, 1982; Shapiro \& ten Berge, 2000).

The maximal reliability, denoted by $\lambda_{\max }$, has recently been focused on (Li, Rosenthal \& Rubin, 1996; Li, 1997; Drewes, 2000; Hancock \& Mueller, 2001; Yuan \& Bentler, 2002; Raykov, 2004, 2005; Raykov \& Penev, 2006), where maximization is made with respect to the weights $\mathbf{w}^{*}=\left(w_{1}^{*}, \ldots, w_{p}^{*}\right)^{\prime}$ for observed variables. Assume that the congeneric or one-factor model holds:

$$
\boldsymbol{\Sigma}=\boldsymbol{\lambda}^{*} \boldsymbol{\lambda}^{*^{\prime}}+\mathbf{\Psi}^{*}
$$

where $\lambda^{*}$ is a $p \times 1$ vector of factor loadings. It is easy to derive the optimal weights as $\mathbf{w}^{*}=\Psi^{*-1} \boldsymbol{\lambda}^{*}$ by using the Lagrange multiplier. The stratified version of $\lambda_{\max }$ can be obtained as in (7.1). Between-set $\lambda_{\max }\left(\lambda_{\max } \mathrm{T}\right)$ is similarly defined with $\boldsymbol{\Psi}^{*}$ taking a form of $\boldsymbol{\Psi}_{\mathrm{B}}^{*}$ as in (7.2).

When $B=2$, (7.3) reduces to the covariance structure of inter-battery factor analysis (Tucker, 1958). It is known that in this model (7.3) has indeterminacy in that $\boldsymbol{\lambda}^{*}$ can be replaced by $\tilde{\boldsymbol{\lambda}}=\left(k \boldsymbol{\lambda}_{1}^{* \prime}, k^{-1} \boldsymbol{\lambda}_{2}^{* \prime}\right)^{\prime}(k>0)$ with $\boldsymbol{\lambda}^{*}=\left(\boldsymbol{\lambda}_{1}^{* \prime}, \boldsymbol{\lambda}_{2}^{* \prime}\right)^{\prime}$ and $\boldsymbol{\Psi}^{*}$ by

$$
\tilde{\boldsymbol{\Psi}}=\mathbf{\Psi}^{*}+\left[\begin{array}{lc}
\left(1-k^{2}\right) \boldsymbol{\lambda}_{1}^{*} \boldsymbol{\lambda}_{1}^{* \prime} & O \\
O & \left(1-k^{-2}\right) \boldsymbol{\lambda}_{2}^{*} \boldsymbol{\lambda}_{2}^{* \prime}
\end{array}\right] \equiv\left[\begin{array}{cc}
\tilde{\boldsymbol{\Psi}}_{11} & O \\
O & \tilde{\boldsymbol{\Psi}}_{22}
\end{array}\right]
$$

as long as $\tilde{\boldsymbol{\Psi}}$ is nonnegative definite. From these results, we see that the reliability depends on $k$, that is

$$
\rho_{X X^{\prime}}=\left(\mathbf{1}_{p}^{\prime} \boldsymbol{\lambda}^{*}\right)^{2} / \sigma^{2}=\left(\mathbf{1}_{p_{1}}^{\prime} \boldsymbol{\lambda}_{1}^{*}+\mathbf{1}_{p_{2}}^{\prime} \boldsymbol{\lambda}_{2}^{*}\right)^{2} / \sigma^{2} \leq \tilde{\rho}_{X X^{\prime}}=\left(\tilde{k} \mathbf{1}_{p_{1}}^{\prime} \boldsymbol{\lambda}_{1}^{*}+\tilde{k}^{-1} \mathbf{1}_{p_{2}}^{\prime} \boldsymbol{\lambda}_{2}^{*}\right)^{2} / \sigma^{2},
$$

where $k=\tilde{k}$ gives the greatest $\rho_{X X^{\prime}}\left(\tilde{\rho}_{X X^{\prime}}\right)$ for given $\boldsymbol{\lambda}^{*}$ over $k>0$ with $\tilde{\boldsymbol{\Psi}}$ being nonnegative definite. Note that $\tilde{k}$ is attained when $k$ is the largest on condition that $\tilde{\boldsymbol{\Psi}}_{11}$ be nonnegative definite or when $k$ is the smallest on condition that $\tilde{\boldsymbol{\Psi}}_{22}$ be nonnegative definite. Fortunately, it is known that as long as $\boldsymbol{\Sigma}$ is positive definite, nonnegative definite 
$\tilde{\boldsymbol{\Psi}}_{11}$ and $\tilde{\boldsymbol{\Psi}}_{22}$ exist (Rao, 1973, Subsection 8f.3; Wegelin et al, 2006).

\section{Appendix 1. The partial derivatives}

Let $\partial \hat{\lambda} /\left.\partial s_{i j}\right|_{\mathbf{s}=\boldsymbol{\sigma}}=\partial \lambda / \partial \sigma_{i j}$ for simplicity of notation with similar expressions for other partial derivatives evaluated at the population values. Denote $\partial \lambda / \partial \sigma_{I_{i} J_{j}}$, $\partial^{2} \lambda / \partial \sigma_{I_{i} J_{j}} \partial \sigma_{K_{k} L_{l}}$ and $\partial^{3} \lambda / \partial \sigma_{I_{i} J_{j}} \partial \sigma_{K_{k} L_{l}} \partial \sigma_{G_{g} H_{h}}$ by $\dot{\lambda}, \ddot{\lambda}$ and $\dddot{\lambda}$, respectively.

(a) $\lambda_{3 \mathrm{~S}}$ (note that $\lambda_{3}$ is a special case of $\lambda_{3 \mathrm{~S}}$ with $B=1$ )

$$
\dot{\lambda}_{3 \mathrm{~S}}=\frac{2-\delta_{I_{i} J_{j}}}{\sigma^{2}}\left\{1-\delta_{I J}+\delta_{I J}\left(1-\delta_{i j}\right) \frac{p_{I}}{p_{I}-1}-\lambda_{3 \mathrm{~S}}\right\}
$$

where $\delta_{i j}$ is the Kronecker delta. The second and third partial derivatives are recursively obtained as

$$
\begin{aligned}
& \ddot{\lambda}_{3 S}=-\frac{1}{\sigma^{2}}\left\{\left(2-\delta_{K_{k} L_{l}}\right) \frac{\partial \lambda_{3 S}}{\partial \sigma_{I_{i} J_{j}}}+\left(2-\delta_{I_{i} J_{j}}\right) \frac{\partial \lambda_{3 S}}{\partial \sigma_{K_{k} L_{l}}}\right\}, \\
& \dddot{\lambda}_{3 S}=-\frac{1}{\sigma^{2}}\left\{\left(2-\delta_{I_{i} J_{j}}\right) \frac{\partial^{2} \lambda_{3 S}}{\partial \sigma_{K_{k} L_{l}} \partial \sigma_{G_{g} H_{h}}}+\left(2-\delta_{K_{k} L_{l}}\right) \frac{\partial^{2} \lambda_{3 S}}{\partial \sigma_{I_{i} J_{j}} \partial \sigma_{G_{g} H_{h}}}+\left(2-\delta_{G_{g} H_{h}}\right) \frac{\partial^{2} \lambda_{3 S}}{\partial \sigma_{I_{i} J_{j}} \partial \sigma_{K_{k} L_{l}}}\right\} \\
& \quad\left(p \geq I_{i} \geq J_{j} \geq 1 ; p \geq K_{k} \geq L_{l} \geq 1 ; p \geq G_{g} \geq H_{h} \geq 1\right) .
\end{aligned}
$$

(b) $\lambda_{3 \mathrm{~T}}$

$$
\dot{\lambda}_{3 \mathrm{~T}}=\frac{2-\delta_{I_{i} J_{j}}}{\sigma^{2}}\left\{\frac{p^{2}}{p^{2}-\sum_{I=1}^{B} p_{I}^{2}}\left(1-\delta_{I J}\right)-\lambda_{3 \mathrm{~T}}\right\} \quad\left(p \geq I_{i} \geq J_{j} \geq 1\right) .
$$

The second and third partial derivatives are the same, in form, as those of $\lambda_{3 S}$. That is, the results are obtained by simply replacing $\lambda_{3 \mathrm{~S}}$ by $\lambda_{3 \mathrm{~T}}$ in (a).

(c) $\lambda_{2 \mathrm{~S}}\left(\lambda_{2}\right)$

$$
\begin{aligned}
\dot{\lambda}_{2 S}= & \frac{2-\delta_{I_{i} J_{j}}}{\sigma^{2}}\left[1-\delta_{I J}+\delta_{I J}\left(1-\delta_{i j}\right)\left\{1+\left(\frac{p_{I}}{p_{I}-1}\right)^{1 / 2} \sigma_{I_{i} I_{j}}\left(\sum_{a \neq b} \sigma_{I_{a} I_{b}}^{2}\right)^{-1 / 2}\right\}-\lambda_{2 \mathrm{~S}}\right], \\
\ddot{\lambda}_{2 S}= & -\frac{1}{\sigma^{2}}\left[\left(2-\delta_{K_{k} L_{l}}\right) \frac{\partial \lambda_{2 \mathrm{~S}}}{\partial \sigma_{I_{i} J_{j}}}+\left(2-\delta_{I_{i} J_{j}}\right) \frac{\partial \lambda_{2 S}}{\partial \sigma_{K_{k} L_{l}}}-2 \delta_{I J K L}\left(1-\delta_{i j}\right)\right. \\
& \left.\times\left(1-\delta_{k l}\right)\left(\frac{p_{I}}{p_{I}-1}\right)^{1 / 2}\left\{\delta_{i k} \delta_{j l}\left(\sum_{a \neq b} \sigma_{I_{a} I_{b}}^{2}\right)^{-1 / 2}-2 \sigma_{I_{i} I_{j}} \sigma_{I_{k} I_{l}}\left(\sum_{a \neq b} \sigma_{I_{a} I_{b}}^{2}\right)^{-3 / 2}\right\}\right],
\end{aligned}
$$

where $\delta_{A B \ldots F}=\delta_{A B} \delta_{B C} \ldots \delta_{E F}$, and 


$$
\begin{aligned}
& \dddot{\lambda}_{2 S}=-\frac{1}{\sigma^{2}}\left[\left(2-\delta_{I_{i} J_{j}}\right) \frac{\partial^{2} \lambda_{2 S}}{\partial \sigma_{K_{k} L_{l}} \partial \sigma_{G_{g} H_{h}}}+\left(2-\delta_{K_{k} L_{l}}\right) \frac{\partial^{2} \lambda_{2 S}}{\partial \sigma_{I_{i} J_{j}} \partial \sigma_{G_{g} H_{h}}}\right. \\
&+\left(2-\delta_{G_{g} H_{h}}\right) \frac{\partial^{2} \lambda_{2 S}}{\partial \sigma_{I_{i} J_{j}} \partial \sigma_{K_{k} L_{l}}}-4 \delta_{I J K L G H}\left(1-\delta_{i j}\right)\left(1-\delta_{k l}\right)\left(1-\delta_{g h}\right)\left(\frac{p_{I}}{p_{I}-1}\right)^{1 / 2} \\
& \times\left\{-\left(\delta_{i k} \delta_{j l} \sigma_{I_{g} I_{h}}+\delta_{i g} \delta_{j h} \sigma_{I_{k} I_{l}}+\delta_{k g} \delta_{l h} \sigma_{I_{i} I_{j}}\right)\left(\sum_{a \neq b} \sigma_{I_{a} I_{b}}^{2}\right)^{-3 / 2}\right. \\
&\left.\left.+6 \sigma_{I_{i} I_{j}} \sigma_{I_{k} I_{l}} \sigma_{I_{g} I_{h}}\left(\sum_{a \neq b} \sigma_{I_{a} I_{b}}^{2}\right)^{-5 / 2}\right\}\right] \\
&\left(p \geq I_{i} \geq J_{j} \geq 1 ; \quad p \geq K_{k} \geq L_{l} \geq 1 ; p \geq G_{g} \geq H_{h} \geq 1\right) .
\end{aligned}
$$

(d) $\lambda_{6 \mathrm{~T}}\left(\lambda_{6}\right)$

$$
\dot{\lambda}_{6 \mathrm{~T}}=\frac{1}{\sigma^{2}}\left\{\left(2-\delta_{I_{i} J_{j}}\right)\left(1-\lambda_{6 \mathrm{~T}}\right)-\sum_{M=1}^{B} \mathbf{1}_{p_{M}}^{\prime} \frac{\partial\left(\boldsymbol{\Sigma}^{M M}\right)^{-1}}{\partial \sigma_{I_{i} J_{j}}} \mathbf{1}_{p_{M}}\right\}
$$

with $\frac{\partial\left(\boldsymbol{\Sigma}^{M M}\right)^{-1}}{\partial \sigma_{I_{i} J_{j}}}=\frac{2-\delta_{I_{i} J_{j}}}{2}\left(\boldsymbol{\Sigma}^{M M}\right)^{-1}\left(\boldsymbol{\Sigma}^{M I_{i}} \boldsymbol{\Sigma}^{J_{j} M}+\boldsymbol{\Sigma}^{M J_{j}} \boldsymbol{\Sigma}^{I_{i} M}\right)\left(\boldsymbol{\Sigma}^{M M}\right)^{-1}$,

where $\boldsymbol{\Sigma}^{M I_{i}}$ with $\boldsymbol{\Sigma}^{I_{i} M}=\left(\boldsymbol{\Sigma}^{M I_{i}}\right)^{\prime}$ is the $p_{M} \times 1$ vector consisting of the elements of the rows for the $M$-th set and the $I_{i}$-th column of $\boldsymbol{\Sigma}$,

$$
\ddot{\lambda}_{6 \mathrm{~T}}=-\frac{1}{\sigma^{2}}\left\{\left(2-\delta_{I_{i} J_{j}}\right) \frac{\partial \lambda_{6 \mathrm{~T}}}{\partial \sigma_{K_{k} L_{l}}}+\left(2-\delta_{K_{k} L_{l}}\right) \frac{\partial \lambda_{6 \mathrm{~T}}}{\partial \sigma_{I_{i} J_{j}}}+\sum_{M=1}^{B} \mathbf{1}_{p_{M}}^{\prime} \frac{\partial^{2}\left(\boldsymbol{\Sigma}^{M M}\right)^{-1}}{\partial \sigma_{I_{i} J_{j}} \partial \sigma_{K_{k} L_{l}}} \mathbf{1}_{p_{M}}\right\}
$$

with

$$
\begin{aligned}
\frac{\partial^{2}\left(\boldsymbol{\Sigma}^{M M}\right)^{-1}}{\partial \sigma_{I_{i} J_{j}} \partial \sigma_{K_{k} L_{l}}}= & \frac{\partial\left(\boldsymbol{\Sigma}^{M M}\right)^{-1}}{\partial \sigma_{I_{i} J_{j}}} \boldsymbol{\Sigma}^{M M} \frac{\partial\left(\boldsymbol{\Sigma}^{M M}\right)^{-1}}{\partial \sigma_{K_{k} L_{l}}}+\frac{\partial\left(\boldsymbol{\Sigma}^{M M}\right)^{-1}}{\partial \sigma_{K_{k} L_{l}}} \boldsymbol{\Sigma}^{M M} \frac{\partial\left(\boldsymbol{\Sigma}^{M M}\right)^{-1}}{\partial \sigma_{I_{i} J_{j}}} \\
& -\frac{1}{4}\left(2-\delta_{I_{i} J_{j}}\right)\left(2-\delta_{K_{k} L_{l}}\right)\left(\boldsymbol{\Sigma}^{M M}\right)^{-1}\left(\sum^{8} \boldsymbol{\Sigma}^{M I_{i}} \sigma^{J_{j} K_{k}} \boldsymbol{\Sigma}^{L_{l} M}\right)\left(\boldsymbol{\Sigma}^{M M}\right)^{-1}
\end{aligned}
$$

and

$$
\begin{aligned}
\dddot{\lambda}_{6 \mathrm{~T}}= & -\frac{1}{\sigma^{2}}\left\{\left(2-\delta_{I_{i} J_{j}}\right) \frac{\partial^{2} \lambda_{6 \mathrm{~T}}}{\partial \sigma_{K_{k} L_{l}} \partial \sigma_{G_{g} H_{h}}}+\left(2-\delta_{K_{k} L_{l}}\right) \frac{\partial^{2} \lambda_{6 \mathrm{~T}}}{\partial \sigma_{I_{i} J_{j}} \partial \sigma_{G_{g} H_{h}}}\right. \\
& \left.+\left(2-\delta_{G_{g} H_{h}}\right) \frac{\partial^{2} \lambda_{6 \mathrm{~T}}}{\partial \sigma_{I_{i} J_{j}} \partial \sigma_{K_{k} L_{l}}}+\sum_{M=1}^{B} \mathbf{1}_{p_{M}}^{\prime} \frac{\partial^{3}\left(\boldsymbol{\Sigma}^{M M}\right)^{-1}}{\partial \sigma_{I_{i} J_{j}} \partial \sigma_{K_{k} L_{l}} \partial \sigma_{G_{g} H_{h}}} \mathbf{1}_{p_{M}}\right\}
\end{aligned}
$$

with

$$
\begin{aligned}
& \frac{\partial^{3}\left(\boldsymbol{\Sigma}^{M M}\right)^{-1}}{\partial \sigma_{I_{i} J_{j}} \partial \sigma_{K_{k} L_{l}} \partial \sigma_{G_{g} H_{h}}}=\frac{\partial^{2}\left(\boldsymbol{\Sigma}^{M M}\right)^{-1}}{\partial \sigma_{I_{i} J_{j}} \partial \sigma_{G_{g} H_{h}}} \boldsymbol{\Sigma}^{M M} \frac{\partial\left(\boldsymbol{\Sigma}^{M M}\right)^{-1}}{\partial \sigma_{K_{k} L_{l}}} \\
& +\frac{\partial\left(\boldsymbol{\Sigma}^{M M}\right)^{-1}}{\partial \sigma_{I_{i} J_{j}}} \boldsymbol{\Sigma}^{M M} \frac{\partial^{2}\left(\boldsymbol{\Sigma}^{M M}\right)^{-1}}{\partial \sigma_{K_{k} L_{l}} \partial \sigma_{G_{g} H_{h}}}+\frac{\partial^{2}\left(\boldsymbol{\Sigma}^{M M}\right)^{-1}}{\partial \sigma_{K_{k} L_{l}} \partial \sigma_{G_{g} H_{h}}} \boldsymbol{\Sigma}^{M M} \frac{\partial\left(\boldsymbol{\Sigma}^{M M}\right)^{-1}}{\partial \sigma_{I_{i} J_{j}}}
\end{aligned}
$$




$$
\begin{aligned}
& +\frac{\partial\left(\boldsymbol{\Sigma}^{M M}\right)^{-1}}{\partial \sigma_{K_{k} L_{l}}} \boldsymbol{\Sigma}^{M M} \frac{\partial^{2}\left(\boldsymbol{\Sigma}^{M M}\right)^{-1}}{\partial \sigma_{I_{i} J_{j}} \partial \sigma_{G_{g} H_{h}}}-\frac{\partial\left(\boldsymbol{\Sigma}^{M M}\right)^{-1}}{\partial \sigma_{I_{i} J_{j}}} \boldsymbol{\Sigma}^{M M} \frac{\partial\left(\boldsymbol{\Sigma}^{M M}\right)^{-1}}{\partial \sigma_{G_{g} H_{h}}} \boldsymbol{\Sigma}^{M M} \frac{\partial\left(\boldsymbol{\Sigma}^{M M}\right)^{-1}}{\partial \sigma_{K_{k} L_{l}}} \\
& -\frac{\partial\left(\boldsymbol{\Sigma}^{M M}\right)^{-1}}{\partial \sigma_{K_{k} L_{l}}} \boldsymbol{\Sigma}^{M M} \frac{\partial\left(\boldsymbol{\Sigma}^{M M}\right)^{-1}}{\partial \sigma_{G_{g} H_{h}}} \boldsymbol{\Sigma}^{M M} \frac{\partial\left(\boldsymbol{\Sigma}^{M M}\right)^{-1}}{\partial \sigma_{I_{i} J_{j}}} \\
& -\frac{1}{4}\left(2-\delta_{I_{i} J_{j}}\right)\left(2-\delta_{K_{k} L_{l}}\right)\left\{\frac{\partial\left(\boldsymbol{\Sigma}^{M M}\right)^{-1}}{\partial \sigma_{G_{g} H_{h}}}\left(\sum^{8} \boldsymbol{\Sigma}^{M I_{i}} \sigma^{J_{j} K_{k}} \boldsymbol{\Sigma}^{L_{l} M}\right)\left(\boldsymbol{\Sigma}^{M M}\right)^{-1}\right. \\
& +\left(\boldsymbol{\Sigma}^{M M}\right)^{-1}\left(\sum^{8} \boldsymbol{\Sigma}^{M I_{i}} \sigma^{J_{j} K_{k}} \boldsymbol{\Sigma}^{L_{l} M}\right) \frac{\partial\left(\boldsymbol{\Sigma}^{M M}\right)^{-1}}{\partial \sigma_{G_{g} H_{h}}} \\
& \left.-\frac{2-\delta_{G_{g} H_{h}}}{2}\left(\boldsymbol{\Sigma}^{M M}\right)^{-1}\left(\sum^{48} \boldsymbol{\Sigma}^{M I_{i}} \sigma^{J_{j} K_{k}} \sigma^{L_{l} G_{g}} \boldsymbol{\Sigma}^{H_{h} M}\right)\left(\boldsymbol{\Sigma}^{M M}\right)^{-1}\right\} \\
& =\sum^{3}\left(\frac{\partial\left(\boldsymbol{\Sigma}^{M M}\right)^{-1}}{\partial \sigma_{I_{i} J_{j}}} \boldsymbol{\Sigma}^{M M} \frac{\partial^{2}\left(\boldsymbol{\Sigma}^{M M}\right)^{-1}}{\partial \sigma_{K_{k} L_{l}} \partial \sigma_{G_{g} H_{h}}}+\frac{\partial^{2}\left(\boldsymbol{\Sigma}^{M M}\right)^{-1}}{\partial \sigma_{K_{k} L_{l}} \partial \sigma_{G_{g} H_{h}}} \boldsymbol{\Sigma}^{M M} \frac{\partial\left(\boldsymbol{\Sigma}^{M M}\right)^{-1}}{\partial \sigma_{I_{i} J_{j}}}\right) \\
& -\sum^{6} \frac{\partial\left(\boldsymbol{\Sigma}^{M M}\right)^{-1}}{\partial \sigma_{I_{i} J_{j}}} \boldsymbol{\Sigma}^{M M} \frac{\partial\left(\boldsymbol{\Sigma}^{M M}\right)^{-1}}{\partial \sigma_{K_{k} L_{l}}} \boldsymbol{\Sigma}^{M M} \frac{\partial\left(\boldsymbol{\Sigma}^{M M}\right)^{-1}}{\partial \sigma_{G_{g} H_{h}}}+\frac{1}{8}\left(2-\delta_{I_{i} J_{j}}\right)\left(2-\delta_{K_{k} L_{l}}\right) \\
& \times\left(2-\delta_{G_{g} H_{h}}\right)\left(\boldsymbol{\Sigma}^{M M}\right)^{-1}\left(\sum^{48} \boldsymbol{\Sigma}^{M I_{i}} \sigma^{J_{j} K_{k}} \sigma^{L_{l} G_{g}} \boldsymbol{\Sigma}^{H_{h} M}\right)\left(\boldsymbol{\Sigma}^{M M}\right)^{-1} \\
& \left(p \geq I_{i} \geq J_{j} \geq 1 ; \quad p \geq K_{k} \geq L_{l} \geq 1 ; \quad p \geq G_{g} \geq H_{h} \geq 1\right),
\end{aligned}
$$

where $\sum^{k}$ denotes the sum of $k$ terms with similar patterns.

(e) $\eta$ 's

The partial derivatives of the coefficients with weights $\mathbf{w}=\mathbf{1}_{p}$ for standardized variables are obtained by using the results for unstandardized variables:

$$
\begin{gathered}
\frac{\partial \eta}{\partial \sigma_{a b}}=\sum_{i>j} \frac{\partial \eta}{\partial \rho_{i j}} \frac{\partial \rho_{i j}}{\partial \sigma_{a b}}, \frac{\partial^{2} \eta}{\partial \sigma_{a b} \partial \sigma_{c d}}=\sum_{i>j}\left(\sum_{k>l} \frac{\partial^{2} \eta}{\partial \rho_{i j} \partial \rho_{k l}} \frac{\partial \rho_{i j}}{\partial \sigma_{a b}} \frac{\partial \rho_{k l}}{\partial \sigma_{c d}}+\frac{\partial \eta}{\partial \rho_{i j}} \frac{\partial^{2} \rho_{i j}}{\partial \sigma_{a b} \partial \sigma_{c d}}\right), \\
\frac{\partial^{3} \eta}{\partial \sigma_{a b} \partial \sigma_{c d} \partial \sigma_{e f}}=\sum_{i>j}\left\{\sum _ { k > l } \left(\sum_{g>h} \frac{\partial^{3} \eta}{\partial \rho_{i j} \partial \rho_{k l} \partial \rho_{g h}} \frac{\partial \rho_{i j}}{\partial \sigma_{a b}} \frac{\partial \rho_{k l}}{\partial \sigma_{c d}} \frac{\partial \rho_{g h}}{\partial \sigma_{e f}}\right.\right. \\
\left.\left.+\sum_{(a b, c d, e f)}^{3} \frac{\partial^{2} \eta}{\partial \rho_{i j} \partial \rho_{k l}} \frac{\partial \rho_{i j}}{\partial \sigma_{a b}} \frac{\partial^{2} \rho_{k l}}{\partial \sigma_{c d} \partial \sigma_{e f}}\right)+\frac{\partial \eta}{\partial \rho_{i j}} \frac{\partial^{3} \rho_{i j}}{\partial \sigma_{a b} \partial \sigma_{c d} \partial \sigma_{e f}}\right\}, \\
(p \geq a \geq b \geq 1 ; p \geq c \geq d \geq 1 ; p \geq e \geq f \geq 1),
\end{gathered}
$$

where $\sum_{i>j}$ denotes $\sum_{p \geq i>j \geq 1}$. The partial derivatives of $\eta$ with respect to $\rho_{i j}$ 's, in form, are given from those of $\lambda$ with respect to $\sigma_{i j}$ 's evaluated at $\boldsymbol{\sigma}=\boldsymbol{\rho}$. The partial derivatives of $\rho_{i j}$ 's with respect to $\sigma_{i j}$ 's are available (Ogasawara, 2006a). When $\mathbf{w} \neq \mathbf{1}_{p}$, 
the values of the above equations are to be multiplied by $w_{a} w_{b}, w_{a} w_{b} w_{c} w_{d}$ and $w_{a} w_{b} w_{c} w_{d} w_{e} w_{f}$, respectively, where the multiplication should be done after obtaining the final results with $\mathbf{w}=\mathbf{1}_{p}$ recursively using lower-order partial derivatives.

\section{Appendix 2. Proof of Theorem 3}

Without loss of generality, let $\boldsymbol{\Sigma}=\mathbf{P}$ with unit population variances and $\mathbf{w}=\mathbf{1}_{p}$ for standardized variables. $\hat{\lambda}$ and $\hat{\eta}$ are written as

$$
\hat{\lambda}=\frac{h(\mathbf{s})}{\mathbf{1}_{p}^{\prime} \mathbf{S} \mathbf{1}_{p}}=\frac{h(\mathbf{s})}{\mathbf{c}^{\prime} \mathbf{s}}, \hat{\eta}=\frac{h(\mathbf{r})}{\mathbf{1}_{p}^{\prime} \mathbf{R} \mathbf{1}_{p}}=\frac{h(\mathbf{r})}{\mathbf{c}^{\prime} \mathbf{r}}, \mathbf{c}=\mathrm{v}\left(2 \mathbf{1}_{p} \mathbf{1}_{p}^{\prime}-\mathbf{I}_{p}\right) .
$$

Firstly, note

$$
\left.\frac{\partial \hat{\lambda}}{\partial s_{i j}}\right|_{\mathbf{s}=\boldsymbol{\sigma}}=\left.\frac{\partial \hat{\eta}}{\partial s_{i j}}\right|_{\mathbf{s}=\boldsymbol{\sigma}}=\left\{\left.\frac{\partial h(\mathbf{s})}{\partial \mathbf{s}^{\prime}}\right|_{\mathbf{s}=\boldsymbol{\sigma}} \mathrm{v}\left(\mathbf{E}_{i j}+\mathbf{E}_{j i}\right)-2 \lambda\right\} \frac{1}{\mathbf{c}^{\prime} \boldsymbol{\sigma}} \quad(p \geq i>j \geq 1)
$$

due to $\boldsymbol{\sigma}=\boldsymbol{\rho}$, where $\mathbf{E}_{i j}$ is the matrix of an appropriate size whose $(i, j)$ th element is 1 with other elements 0 . On the other hand,

$$
\begin{aligned}
\frac{\partial \hat{\lambda}}{\partial s_{i i}}= & \left(\frac{\partial h(\mathbf{s})}{\partial \mathbf{s}^{\prime}} \mathrm{v}\left(\mathbf{E}_{i i}\right)-\hat{\lambda}\right) \frac{1}{\mathbf{c}^{\prime} \mathbf{s}} \\
\frac{\partial \hat{\eta}}{\partial s_{i i}}= & \frac{\partial h(\mathbf{r})}{\partial \mathbf{r}^{\prime}} \frac{\partial \mathbf{r}}{\partial s_{i i}} \frac{1}{\mathbf{c}^{\prime} \mathbf{r}}-\frac{\hat{\eta}}{\mathbf{c}^{\prime} \mathbf{r}} \frac{\partial \mathbf{c}^{\prime} \mathbf{r}}{\partial s_{i i}} \\
= & \frac{\partial h(\mathbf{r})}{\partial \mathbf{r}^{\prime}} \mathrm{v}\left(\mathbf{E}_{i i} r_{i i}-\frac{1}{2} \mathbf{E}_{i i} \mathbf{R}-\frac{1}{2} \mathbf{R E}_{i i}\right) \frac{s_{i i}^{-1}}{\mathbf{c}^{\prime} \mathbf{r}} \\
& -\frac{\hat{\eta}}{\mathbf{c}^{\prime} \mathbf{r}} \mathbf{1}_{p}^{\prime}\left(\mathbf{E}_{i i} r_{i i}-\frac{1}{2} \mathbf{E}_{i i} \mathbf{R}-\frac{1}{2} \mathbf{R} \mathbf{E}_{i i}\right) \mathbf{1}_{p} s_{i i}^{-1} \quad(i=1, \ldots, p) .
\end{aligned}
$$

Evaluating the difference of the above two equations at the population values, it follows that

$$
\begin{aligned}
\left.\frac{\partial \hat{\eta}}{\partial s_{i i}}\right|_{\mathbf{s}=\boldsymbol{\rho}} & =\left.\frac{\partial \hat{\lambda}}{\partial s_{i i}}\right|_{\mathbf{s}=\boldsymbol{\rho}}-\left.\frac{1}{2} \frac{\partial h(\mathbf{r})}{\partial \mathbf{r}^{\prime}}\right|_{\mathbf{r}=\boldsymbol{\rho} \mathrm{v}\left(\mathbf{E}_{i i} \mathbf{P}+\mathbf{P} \mathbf{E}_{i i}\right) \frac{1}{\mathbf{c}^{\prime} \boldsymbol{\rho}}+\frac{\eta}{\mathbf{c}^{\prime} \boldsymbol{\rho}} \mathbf{1}_{p}^{\prime} \boldsymbol{\rho}_{\cdot i}} \\
& =\left.\frac{\partial \hat{\lambda}}{\partial s_{i i}}\right|_{\mathbf{s}=\boldsymbol{\rho}}-\frac{1}{2 \mathbf{c}^{\prime} \boldsymbol{\rho}}\left\{\left.\frac{\partial h(\mathbf{r})}{\partial \mathbf{r}_{\cdot i}^{\prime}}\right|_{\mathbf{r}=\boldsymbol{\rho}} \boldsymbol{\rho}_{\cdot i}+\left.\frac{\partial h(\mathbf{r})}{\partial r_{i i}}\right|_{\mathbf{r}=\boldsymbol{\rho}}-2 \eta \mathbf{1}_{p}^{\prime} \boldsymbol{\rho} \cdot i\right\} \quad(i=1, \ldots, p),
\end{aligned}
$$

which yields $\partial \hat{\eta} /\left.\partial \mathbf{s}\right|_{\mathbf{s}=\boldsymbol{\rho}}=\partial \hat{\lambda} /\left.\partial \mathbf{s}\right|_{\mathbf{s}=\boldsymbol{\rho}}$ when (5.1) holds for $i=1, \ldots, p$. 


\section{REFERENCES}

Bentler, P.M. (1972). A lower-bound method for the dimension-free measurement of internal consistency. Social Science Research, 1, 343-357

Bentler, P.M., \& Woodward, J.A. (1980). Inequalities among lower bounds to reliability: With applications to test construction and factor analysis. Psychometrika, 45, 249-267.

Browne, M.W. (1992). Circumplex models for correlation matrices. Psychometrika, 57, 469-497.

Cronbach, L.J. (1951). Coefficient alpha and the internal structure of tests. Psychometrika, 16, $297-334$.

Cronbach, L.J. (1988). Internal consistency of tests: Analyses old and new. Psychometrika, 56, 63-70.

Cronbach, L.J., Schönemann, P., \& McKie, D. (1965). Alpha coefficients for stratified-parallel tests. Educational and Psychological Measurement, 25, 291-312.

Drewes, D.W. (2000). Beyond the Spearman-Brown: A structural approach to maximum reliability. Psychological Methods, 5, 214-227.

Feldt, L.S. (1965). The approximate sampling distribution of Kuder-Richardson reliability coefficient twenty. Psychometrika, 30, 357-370.

Feldt, L.S., \& Brennan, R.L. (1989). Reliability. In R.L. Linn (Ed.), Educational measurement (3rd ed., pp.105-146). New York: Macmillan.

Feldt, L.S., Woodruff, D.J., \& Salih, F.A. (1987). Statistical inference for coefficient alpha. Applied Psychological Measurement, 11, 93-103.

Guttman, L. (1945). A basis for analyzing test-retest reliability. Psychometrika, 10, 255-282.

Guttman, L. (1954). A new approach to factor analysis: The radex. In P.F. Lazarsfeld (Ed.), Mathematical thinking in the social sciences (pp. 258-348). New York: Columbia University Press.

Hall, P. (1992). On the removal of skewness by transformation. Journal of the Royal Statistical Society, B, 54, 221-228.

Hancock, G.R., \& Mueller, R.O. (2001). Rethinking construct reliability within latent variable systems. In R. Cudeck, S. du Toit \& D. Sörbom (Eds.), Structural equation modeling: Present and future (pp.195-216). Lincolnwood, IL: Scientific Software International.

Hayashi, K., \& Kamata, A. (2005). A note on the estimator of the alpha coefficient for standardized variables under normality. Psychometrika, 70, 579-589.

Jackson, P.H., \& Agunwanba, C.C. (1977). Lower bounds for the reliability of the total score on a test composed of non-homogeneous items: I: Algebraic lower bounds. Psychometrika, 42, $567-578$.

Jöreskog, K.G., \& Sörbom, D. (1976). Statistical models and methods for test-retest situations. In D.N.M. de Gruijter \& L.J. Th. van der Kamp (Eds.), Advances in psychological and educational measurement (pp.135-157). New York: Wiley.

Kistner, E.O., \& Muller, K.E. (2004). Exact distributions of intraclass correlation and Cronbach's alpha with Gaussian data and general covariance. Psychometrika, 69, 459-474.

Komaroff, E. (1997). Effect of simultaneous violations of essential $\tau$-equivalence and uncorrelated error on coefficient $\alpha$. Applied Psychological Measurement, 21, 337-348.

Kristof, W. (1963). The statistical theory of speeded-up reliability coefficients when a test has been divided into several equivalent parts. Psychometrika, 28, 221-238.

Li, H. (1997). A unifying expression for the maximal reliability of a linear composite. Psychometrika, 62, 245-249.

Li, H., Rosental, R., \& Rubin, D.B. (1996). Reliability of measurement in psychology: From Spearman-Brown to maximal reliability. Psychological Methods, 1, 98-107

Lord, F.M., \& Novick, M.R. (1968). Statistical theories of mental test scores. Reading, MA: Addison-Wesley. 
McDonald, R.P. (1970). The theoretical foundations of principal factor analysis, canonical factor analysis and alpha factor analysis. British Journal of Mathematical and Statistical Psychology, 23, 1-21.

McDonald, R.P. (1999). Test theory: A unified treatment. Mahwah, NJ: Erlbaum.

Novick, M.R., \& Lewis, C. (1967). Coefficient alpha and the reliability of composite measurements. Psychometrika, 32, 1-13.

Ogasawara, H. (2006a). Asymptotic expansion of the sample correlation coefficient under nonnormality. Computational Statistics and Data Analysis, 50, 891-910.

Ogasawara, H. (2006b). Approximations to the distribution of the sample coefficient alpha under nonnormality. Behaviormetrika, 33, 3-26.

Ogasawara, H. (2007a). Higher-order approximations to the distributions of fit indexes under fixed alternatives in structural equation models. Psychometrika, 72, 227-243.

Ogasawara, H. (2007b). Asymptotic expansion of the distributions of the estimators in factor analysis under nonnormality. British Journal of Mathematical and Statistical Psychology, 60, 395-420.

Osburn, H.G. (2000). Coefficient alpha and related internal consistency reliability coefficients. Psychological Methods, 5, 343-355.

Rajaratnam, N., Cronbach, L.J., \& Gleser, G.C. (1965). Generalizability of stratified-parallel tests. Psychometrika, 30, 39-56.

Rao, C.R. (1973). Linear statistical inference and its applications (2nd ed.). New York: Wiley.

Raykov, T. (1997a). Scale reliability, Cronbach's coefficient alpha, and violations of essential tau-equivalence with fixed congeneric components. Multivariate Behavioral Research, 32, 329-353.

Raykov, T. (1997b). Estimation of composite reliability for congeneric measures. Applied Psychological Measurement, 21, 173-184.

Raykov, T. (1998). Coefficient alpha and composite reliability with interrelated nonhomogeneous items. Applied Psychological Measurement, 22, 375-385.

Raykov, T. (2001). Bias of coefficient $\alpha$ for fixed congeneric measures with correlated errors. Applied Psychological Measurement, 25, 69-76.

Raykov, T. (2002). Analytic estimation of standard error and confidence interval for scale reliability. Multivariate Behavioral Research, 37, 89-103.

Raykov, T. (2004). Estimation of maximal reliability: A note on a covariance structure modeling approach. British Journal of Mathematical and Statistical Psychology, 57, 21-27.

Raykov, T. (2005). Studying group and time invariance in maximal reliability for multiplecomponent measuring instruments via covariance structure modeling. British Journal of Mathematical and Statistical Psychology, 58, 301-317.

Raykov, T., \& Marcoulides, G.A. (2004). Using the delta method for approximate interval estimation of parameter functions in SEM. Structural Equation Modeling, 11, 621-637.

Raykov, T., \& Penev, S. (2006). A direct method for obtaining approximate standard error and confidence interval of maximal reliability for composites with congeneric measures. Multivariate Behavioral Research, 41, 15-28.

Raykov, T., \& Shrout, P.E. (2002). Reliability of scales with general structure: Point and interval estimation using a structural equation modeling approach. Structural Equation Modeling, 9, 195-212.

Shapiro, A. (1982). Rank-reduciability of a symmetric matrix and sampling theory of minimum trace factor analysis. Psychometrika, 47, 187-199.

Shapiro, A., \& ten Berge, J.M.F. (2000). The asymptotic bias of minimum trace factor analysis, with applications to the greatest lower bound to reliability. Psychometrika, 65, 413-425.

Stuart, A., \& Ord, J.K. (1994). Kendall's advanced theory of statistics: Distribution theory (6th ed., Vol.1). London: Arnold. 
ten Berge, J.M.F., Snijders, T.A.B., \& Zegers, F.E. (1981). Computational aspects of the greatest lower bound to the reliability and constrained minimum trace factor analysis. Psychometrika, 46, 201-213.

ten Berge, J.M.F., \& Socan, G. (2004). The greatest lower bound to the reliability of a test and the hypothesis of unidimensionality. Psychometrika, 69, 613-625.

ten Berge, J.M.F., \& Zegers, F.E. (1978). A series of lower bounds to the reliability of a test. Psychometrika, 43, 575-579.

Tucker, L.R. (1958). An inter-battery method of factor analysis. Psychometrika, 23, 111-136.

van Zyl, J.M., Neudecker, H., \& Nel, D.G. (2000). On the distribution of the maximum likelihood estimator of Cronbach's alpha. Psychometrika, 65, 271-280.

Wegelin, J.A., Packer, A., \& Richardson, T.S. (2006). Latent models for cross-covariance. Journal of Multivariate Analysis, 97, 79-102.

Woodhouse, B., \& Jackson, P.H. (1977). Lower bounds for the reliability of the total score on a test composed of non-homogeneous items: II: A search procedure to locate the greatest lower bound. Psychometrika, 42, 579-591.

Yuan, K.-H., \& Bentler, P. (2002). On robustness of the normal-theory based asymptotic distributions of three reliability coefficient estimates. Psychometrika, 67, 251-259.

Zimmerman, D.W., Zumbo, B.D., \& Lalonde, C. (1993). Coefficient alpha as an estimate of test reliability under violation of two assumptions. Educational and Psychological Measurement, 53, 33-49.

Zinbarg, R.E., Revelle, W., Yovel, I., \& Li, W. (2005). Cronbach's $\alpha$, Revelle's $\beta$, and McDonald's $\omega_{H}$ : Their relations with each other and two alternative conceptualizations of reliability. Psychometrika, 70, 123-133. 